\title{
Small-scale turbulence characteristics of two-dimensional bluff body wakes
}

\author{
By R. A. ANTONIA ${ }^{1}, T \cdot \mathrm{ZHOU}^{2}$ AND G. P. ROMANO \\ ${ }^{1}$ Department of Mechanical Engineering, University of Newcastle, NSW, 2308, Australia \\ ${ }^{2}$ Department of Mechanical \& Production Engineering, Nanyang Technological University, \\ Singapore 639798 \\ ${ }^{3}$ Dipartimento di Meccanica e Aeronautica, Universita Degli Studi di Roma "La Sapienza",
} 00184 Rome, Italy

(Received 11 May 2001 and in revised form 30 October 2001)

Measurements have been made in nominally two-dimensional turbulent wakes generated by five different bluff bodies. Each wake has a different level of large-scale organization which is reflected in different amounts of large-scale anisotropy. Structure functions of streamwise $(u)$ and lateral $(v)$ velocity fluctuations at approximately the same value of $R_{\lambda}$, the Taylor microscale Reynolds number, indicate that inertial-range scales are significantly affected by the large-scale anisotropy. The effect is greater on $v$ than $u$ and more pronounced for the porous-body wakes than the solid-body wakes. In particular, 'relative' values of the scaling (or power-law) exponents indicate that the magnitude of the transverse exponents can exceed that of the longitudinal ones in the porous-body wakes. This is supported by the inertial-range behaviour of the spectra of $u$ and $v$. The difference between the transverse and longitudinal exponents appears to depend on the large-scale anisotropy of the flow, as measured by the ratio of the variances of $v$ and $u$ and ratio of the integral length scales of $v$ and $u$. The spanwise vorticity spectra are much less affected by the anisotropy than the spectra of $u$ and $v$.

\section{Introduction}

The analytical framework introduced by Kolmogorov $(1941 a, b)$ for describing the properties of small-scale turbulence has had an almost immeasurable impact on turbulence research. There continues to be a strong belief among turbulence researchers that if a theory of turbulence were to emerge, it would most likely relate to the small-scale motion whose features tend to exhibit quasi-universal characteristics. The similarity hypotheses proposed by Kolmogorov (1941a, hereafter K41), provide a description of turbulence in both the dissipation range (DR), where the scales of the motion are such that they may be influenced by the kinematic viscosity $v$ of the fluid, and the inertial range (IR), loosely defined as the range of scales which lies between the smallest length scale of the flow (usually identified with the Kolmogorov length scale $\eta \equiv v^{3 / 4} /\langle\epsilon\rangle^{1 / 4}$, where $\langle\epsilon\rangle$ is the mean energy dissipation rate) and the largest length scale (typically identified with the longitudinal integral length $L_{u}$, defined in $\S 3)$. The first hypothesis in K41 states that, in the DR, the probability density function (p.d.f.) of the velocity increment $\delta \alpha \equiv \alpha(x+r)-\alpha(r)(r$ is the separation between the two points and $\alpha$ stands for $u$ or $v$ or $w$, the velocity fluctuations in the $x$ (longitudinal or streamwise), $y$ (mean shear) or $z$ (spanwise) directions) is uniquely determined by 
$\langle\epsilon\rangle$ and $v$ so that

$$
\left\langle\left(\delta \alpha^{*}\right)^{n}\right\rangle=f_{\alpha n}\left(r^{*}\right),
$$

where the asterisk denotes normalization by $\eta$ and/or $U_{K} \equiv(v\langle\epsilon\rangle)^{1 / 4}$, the Kolmogorov velocity scale. The second hypothesis states that, in the IR $\left(\eta \ll r \ll L_{u}\right)$,

$$
\left\langle\left(\delta \alpha^{*}\right)^{n}\right\rangle=C_{\alpha n} r^{* n / 3},
$$

where $C_{\alpha n}$ are the Kolmogorov 'constants' (which may depend on the flow macrostructure). It is important to stress that (1.1) and (1.2) are underpinned by the (related) assumptions that the Reynolds number is large and the small scales are isotropic. As was noted by Sreenivasan (1999), K41 supposes that the small scales can be understood independently of the large-scale motion which may differ from flow to flow and is likely to be strongly anisotropic.

To account for the small-scale intermittency (or wild and sudden fluctuations in time and space of $\epsilon$ which lead to strong departures of turbulence quantities from a simple Gaussian behaviour), Kolmogorov (1962) and Obukhov (1962) (the combination of these two papers is usually referred to as K62) modified the dimensional arguments of K41 by replacing $\langle\epsilon\rangle$ with $\epsilon_{r}$ where the subscript denotes averaging over a length scale $r$. In K62, (1.2) becomes

$$
\left\langle\left(\delta \alpha^{*}\right)^{n}\right\rangle=C_{\alpha, n}^{+}\left\langle\epsilon_{r}^{* n / 3}\right\rangle r^{* n / 3},
$$

where the superscript + allows a possible distinction between the premultipliers in (1.3) and those in (1.2). K62 admits a power-law variation in the IR provided $\left\langle\epsilon_{r}^{* n / 3}\right\rangle$ has a power-law variation. In this case, $\left\langle\left(\delta \alpha^{*}\right)^{n}\right\rangle$ can be expressed (in the IR) as

$$
\left\langle\left(\delta \alpha^{*}\right)^{n}\right\rangle \sim r^{*} \zeta_{\alpha n} .
$$

While K62 offers advantages over K41, it also detracts from the inherent simplicity of the K41 framework. Regardless of whether one favours K41 over K62 or vice versa, the main assumptions which underpin K41 also apply to K62 and the reality is that they are unlikely to be satisfied in flows normally encountered in the laboratory. It is also dubious whether these assumptions are accurately satisfied in the atmospheric surface layer, despite the relatively large values of $R_{\lambda}$ (a Reynolds number based on $\left\langle u^{2}\right\rangle^{1 / 2}$ and $\lambda$, the longitudinal Taylor microscale); for example, Sreenivasan \& Dhruva (1998) found that, in the atmospheric surface layer with $R_{\lambda} \sim 10^{4}$, unique values of the exponents $\zeta_{\alpha n}$ could not be evaluated unambiguously since the local derivatives of $\left\langle(\delta \alpha)^{n}\right\rangle$, with respect to $r$, continuously decreased with increasing $r$. Notwithstanding the difficulty of determining $\zeta_{\alpha n}$ rigorously, there is significant evidence to suggest that $\zeta_{\alpha n}$ may depend on $R_{\lambda}$ (e.g. Antonia, Pearson \& Zhou 2000), vary from flow to flow (e.g. Pearson 1999), be affected by the presence of a wall (e.g. Antonia, Romano \& Orlandi 1998), or indeed depend on the initial conditions for nominally the same flow (e.g. Antonia \& Pearson 2000; Romano \& Antonia 2001). It is also significant that, for $n=2$, the isotropic equality $\zeta_{u 2}=\zeta_{v 2}=\zeta_{w 2}$ is in general not satisfied by the data (from both measurements and numerical flow simulations). Typically, the magnitudes of the transverse exponents $\zeta_{v n}$ and $\zeta_{w n}$ tend to be smaller than those of the longitudinal exponents $\zeta_{u n}$ (in general, transverse exponents can also be associated with $u$ structure functions but for separations in the transverse directions). The most likely causes for this inequality are

(i) the effect of $R_{\lambda}$,

(ii) the influence of anisotropy,

(iii) the nature of the flow,

(iv) the differences in initial and boundary conditions. 
It has also been suggested that the sources of small-scale intermittency which affect longitudinal and transverse structure functions may be different (e.g. Chen et al. 1997) although the evidence is not entirely conclusive (e.g. Zhou \& Antonia 2000). Ideally, each of the above effects should be studied separately. In practice, this is not always feasible either experimentally or in simulations. Ideally, the study of (i) should be carried out in the same flow (initial and boundary conditions should remain unchanged) in the absence of a mean shear or other sources of anisotropy; extraneous influences, such as that arising from the large-scale intermittency associated with the presence of a turbulent/non-turbulent interface, should be absent. Published data that have been used to study (i) were obtained in different flows (and almost invariable different turbulence intensities) although usually in regions where the mean shear was negligible.

The aim of the present investigation is to examine the dependence of the smallscale motion on initial conditions by focusing on nominally the same flow: a twodimensional wake generated by different bluff bodies. Both porous and impervious bodies were chosen because of the differences in the organized motion that have been observed when the shape and/or porosity of the generator are varied (e.g. Cannon, Champagne \& Glezer 1983; Cimbala, Nagib \& Roshko 1988; Zhou \& Antonia 1994, 1995; Antonia \& Mi 1998) or when, for a fixed shape, the mass injection rate through the downstream end of the generator is varied (Cimbala \& Park 1990; Park \& Cimbala 1991). Although the differences are most pronounced in the near wake, they do not entirely disappear in the far wake. In association with the differences in the large-scale organization, and hence in the manner in which the initial injection of energy takes place, there are differences in the overall or global anisotropy. A measure of this anisotropy is provided by the ratio $\left\langle v^{2}\right\rangle /\left\langle u^{2}\right\rangle$. In the present experiments, a significant variation of this ratio was achieved for nominally the same flow and the same $R_{\lambda}$. The measurements were made only on the wake centreline where the mean shear is zero and the flow is fully turbulent.

Recently, significant attention has been given to the extraction of anisotropic contributions from velocity structure functions obtained both in experiment and numerical simulations (e.g. Arad et al. 1998; Kurien \& Sreenivasan 2000; Biferale \& Vergassola 2001; Biferale \& Toschi 2001). The approach is based on decomposing velocity correlations or structure functions in terms of the irreducible representation of the $\mathrm{SO}(3)$ group of spatial rotation in three dimensions (Arad, L'vov \& Procaccia $1999 b$ ). The isotropic sector of this decomposition is the zero-order term which can be disentangled from the anisotropic part (Arad et al. 1999a). The primary motivation for this approach is the elucidation of the apparent persistence of anisotropic effects on scales in both the inertial and dissipative ranges. Although the approach is quite promising and encouraging results have been obtained (e.g. Kurien \& Sreenivasan 2000; Biferale \& Vergassola 2001), the exact projection via spherical harmonics on each separate sector is not straightforward even for numerical simulation data (e.g. Biferale \& Vergassola 2001). Only incomplete information on the velocity field is available in the present experiments; for this reason, the $\mathrm{SO}(3)$ decomposition has not been used.

\section{Experimental conditions}

In this study, a non-return blower-type wind tunnel with a square $(350 \times 350 \mathrm{~mm})$ $2.4 \mathrm{~m}$ long working section was used. The inclination of the bottom wall of the working section was adjusted in order to maintain a zero streamwise pressure gradient. 


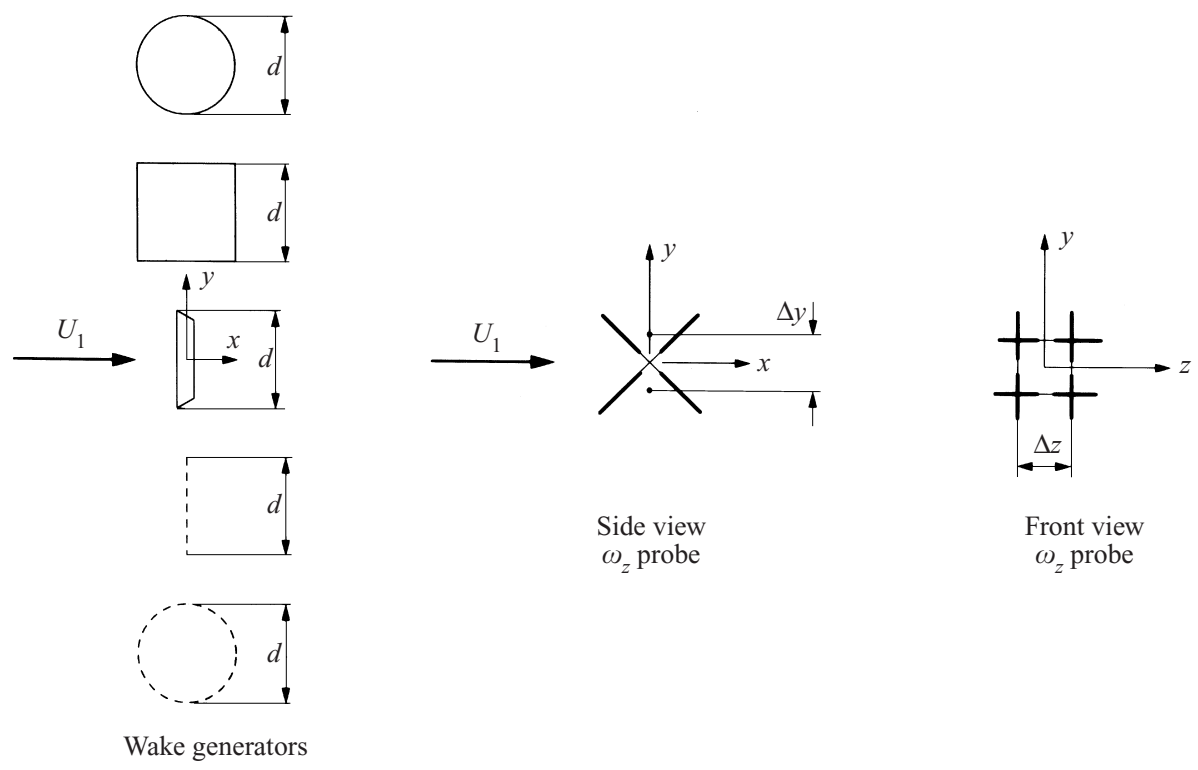

FIGURE 1. Sketch showing arrangement of different wake generators, coordinate axes and spanwise vorticity probe.

Measurements of the spanwise vorticity fluctuation $\omega_{z}$ were made on the centreline of wakes generated by five different bluff bodies, i.e. a solid circular cylinder, a circular cylinder constructed from a screen $(0.5 \mathrm{~mm}$ wire diameter with a mesh size of $1.6 \mathrm{~mm})$ of $54 \%$ solidity, a solid square cylinder, a solid plate placed normal to the flow, and screen strip constructed of the same material as the screen cylinder. In each case (figure 1), the characteristic dimension (height or diameter) $d$ is the same $(25.4 \mathrm{~mm})$. The generators are introduced $20 \mathrm{~cm}$ downstream of the entrance to the working section and span the full width of the working section. The measurement location was at $x / d=70$. While this distance may be adequate for the mean velocity profile to become self-preserving, it is unlikely that the Reynolds stresses are self-preserving there. The choice was thought however to be a reasonable compromise due to the opposing constraints imposed by the finite length of the working section and the need to use a sufficiently large value of $d$ for the Kolmogorov length scale to be sufficiently large. It is well established (e.g. Sreenivasan 1981) that the asymptotic far wake is approached only slowly, the approaching depending on the manner the wake is generated. The intent of the present experiment is to study the effect of different large scale forcings, resulting from different initial conditions, on small-scale statistics at the same $x / d$ and Taylor microscale Reynolds number $R_{\lambda}$. Before measuring the spanwise vorticity fluctuations, systematic measurements with a single hot wire were made to determine the dependence of $R_{\lambda}$ on the mean velocity in each wake. Measurements of $\omega_{3}$ were made at five different values of free-stream velocity for each wake. Details of experimental conditions in each of the wakes are given in table 1 for approximately the same $R_{\lambda}$.

The transverse vorticity component $\omega_{z}$ was obtained using a four-hot-wire vorticity probe (sketched in figure 1). This probe consists of a pair of parallel wires $c$ and $d$ straddling wires $a$ and $b$ of an X-probe. The quantity $\omega_{z}$ can be approximated by

$$
\omega_{z}=\frac{\Delta v}{\Delta x}-\frac{\Delta u}{\Delta y}=-U^{-1} \frac{\Delta v}{\Delta t}-\frac{\Delta u}{\Delta y},
$$




\begin{tabular}{lccccccccccc}
\hline & $\begin{array}{c}U \\
\left(\mathrm{~m} \mathrm{~s}^{-1}\right)\end{array}$ & $\begin{array}{c}u^{\prime} \\
\left(\mathrm{m} \mathrm{s}^{-1}\right)\end{array}$ & $\begin{array}{c}v^{\prime} \\
\left(\mathrm{m} \mathrm{s}^{-1}\right)\end{array}$ & $\begin{array}{c}f_{c} \\
(\mathrm{~Hz})\end{array}$ & $\begin{array}{c}f_{s} \\
(\mathrm{~Hz})\end{array}$ & $\begin{array}{c}f_{K} \\
(\mathrm{~Hz})\end{array}$ & $\begin{array}{c}\langle\epsilon\rangle \dagger \\
\left(\mathrm{m}^{2} \mathrm{~s}^{-3}\right)\end{array}$ & $\begin{array}{c}\eta \\
(\mathrm{mm})\end{array}$ & $R_{\lambda}$ & $\begin{array}{c}U_{K} \\
\left(\mathrm{~m} \mathrm{~s}^{-1}\right)\end{array}$ & $\Delta y^{*}$ \\
Flow & 8.75 & 0.538 & 0.482 & 6300 & 12903 & 7150 & 2.265 & 0.195 & 194 & 0.076 & 3.8 \\
$\mathrm{CC}$ & 8.27 & 0.583 & 0.594 & 6300 & 12903 & 7300 & 3.090 & 0.180 & 195 & 0.082 & 4.2 \\
$\mathrm{P}$ & 7.52 & 0.573 & 0.539 & 6300 & 12903 & 6506 & 2.847 & 0.184 & 196 & 0.080 & 4.2 \\
$\mathrm{SqC}$ & 6.22 & 0.457 & 0.429 & 4000 & 8000 & 4372 & 1.240 & 0.227 & 189 & 0.065 & 3.2 \\
$\mathrm{ScC}$ & 15.09 & 0.861 & 1.008 & 25000 & 50000 & 19290 & 13.602 & 0.125 & 204 & 0.119 & 4.7 \\
$\mathrm{SS}$ & \multicolumn{7}{c}{ Isotropy has been assumed, i.e. $\langle\epsilon\rangle$ was inferred from $\langle\epsilon\rangle_{\text {iso }}=15 v\left\langle(\partial u / \partial x)^{2}\right\rangle$} &
\end{tabular}

TABLE 1. Experimental conditions at $x / d=70$ and nominally the same value of $R_{\lambda}$. CC denotes circular cylinder, $\mathrm{P}$ plate, $\mathrm{SqC}$ square cylinder, $\mathrm{ScC}$ screen cylinder and $\mathrm{SS}$ screen strip.

where $\Delta u$ is the difference between the longitudinal velocity fluctuations from two parallel hot wires which are separated in the $y$-direction; $\Delta v$ is the difference between values of $v$ at the same point in space but separated in time by one sampling time interval $\Delta t\left(\approx f_{s}^{-1} ; f_{s}\right.$ is the sampling frequency) and $U$ is the local streamwise mean velocity. Because the turbulence intensity is relatively small $\left(u_{i}^{\prime} / U_{1} \leqslant 8 \%\right.$, where $u_{i}$ represents $u$ or $v$ or $w$ and the prime denotes the r.m.s. value), the use of Taylor's hypothesis, i.e. $\Delta / \Delta x=-U^{-1} \Delta / \Delta t$ in (2.1), should be satisfactory, especially at small $r$. A forward differencing scheme was used to convert temporal to spatial derivatives, the magnitude of $\Delta x$ being approximately equal to that of $\Delta y$.

The separation $\Delta y$, which was about $0.75 \mathrm{~mm}$, was adequate since it remained in the range $3 \eta-5 \eta$ (e.g. Antonia, Zhu \& Kim 1993; Zhu \& Antonia 1995). This strategy was adopted to try and minimize any noise contamination due to too small a separation and any spectral attenuation due to too large a separation. The wire separation $\Delta z$ between the two inclined wires of the X-probe was about $0.8 \mathrm{~mm}$. Values of $\Delta z^{*}$ are not shown in the table since $\Delta z^{*} \simeq \Delta y^{*}$ in each flow. The included angle for the $\mathrm{X}$-probe was about $105^{\circ}$, large enough to minimize the effect of large velocity cone angles (e.g. Browne, Antonia \& Chua 1989). The probe comprised $2.5 \mu \mathrm{m}$ diameter Wollaston $\mathrm{Pt}-10 \% \mathrm{Rh}$ wires, each etched to an active length of about $0.5 \mathrm{~mm}$. The length to diameter ratio of the wire was about 200 .

The hot wires were operated with in-house constant temperature circuits at an overheat ratio of 1.5. Output signals from the anemometers were passed through buck-and-gain circuits and low-pass filtered at a cut-off frequency $f_{c}$ (see table 1); $f_{c}$ was chosen after examining the spectrum of $\partial u / \partial t$ and identifying the onset of electronic noise (the procedure was similar to that outlined in Antonia, Satyaprakash $\&$ Hussain 1982). The values of $f_{c}$ were generally close to the Kolmogorov frequency $f_{K} \equiv U / 2 \pi \eta$. The filtered signals were then sampled at a frequency of $f_{s} \simeq 2 f_{c}$ into a PC using a 12 bit A/D converter. The sampling period $T_{s}$ is in the range $45-100 \mathrm{~s}$ for each wake. The corresponding number of independent samples $N$ ( $\equiv T_{s} U / 2 L$, e.g. Tennekes \& Lumley 1972) is in the range 3000-19000. These numbers are large enough for the eighth-order velocity structure functions to converge. This was verified by examining the closure of the p.d.f. of $\delta \alpha$, weighted by $|\delta \alpha|^{n}$, for different values of $r$.

\section{Global characteristics of the different wakes}

Before discussing the behaviour of the small scales $(\$ \S 4-7)$, we present here a few results which characterize the global features of the wakes. A measure of the anisotropy of the large scales is provided by the turbulence intensity ratio $\left\langle v^{2}\right\rangle /\left\langle u^{2}\right\rangle$, 


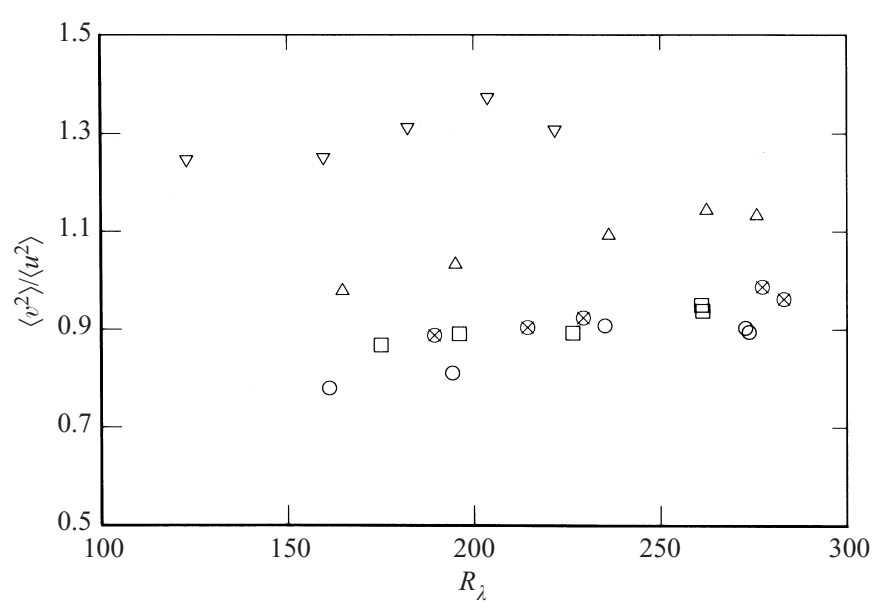

FIGURE 2. Dependence of the Reynolds normal stress ratio $\left\langle v^{2}\right\rangle /\left\langle u^{2}\right\rangle$ on $R_{\lambda}$ in each of the wakes: $\bigcirc$, circular solid cylinder; $\square$, solid square cylinder; $\triangle$, solid normal plate; $\nabla$, screen strip; $\otimes$, circular screen cylinder.

where $\left\langle v^{2}\right\rangle$ and $\left\langle u^{2}\right\rangle$ are the variances of $v$ and $u$. Figure 2 indicates that, in general, the magnitude of this ratio differs significantly from flow to flow. In particular, it is largest for the screen strip and smallest for the circular cylinder. Note that for both the screen strip and the plate, $\left\langle v^{2}\right\rangle$ is larger than $\left\langle u^{2}\right\rangle$, while in the other three wakes, it is $\left\langle u^{2}\right\rangle$ that is larger. The values of $\left\langle v^{2}\right\rangle /\left\langle u^{2}\right\rangle$ are nearly identical for the square cylinder and the screen cylinder. There is a slight tendency for $\left\langle v^{2}\right\rangle /\left\langle u^{2}\right\rangle$ to increase with $R_{\lambda}$ although, arguably, the ratio may become constant at sufficiently large $R_{\lambda}$. Overall, the results of figure 2 imply that, in almost every case, the large scales are likely to exhibit differences, either topologically or in terms of the strength of the vortices (e.g. Cimbala et al. 1988; Zhou \& Antonia 1994). Most of the variation in $\left\langle v^{2}\right\rangle /\left\langle u^{2}\right\rangle$ is caused by changes in $\left\langle v^{2}\right\rangle$ rather than in $\left\langle u^{2}\right\rangle$.

It is well established that $v$ is a more sensitive indicator of the large-scale organization than $u$ (e.g. Papailiou \& Lykoudis 1974). Spectra or, equivalently, correlations can be used to highlight possible differences in this organization. The autocorrelation coefficients $\rho_{\alpha} \equiv\langle\alpha(x) \alpha(x+r)\rangle /\left\langle\alpha^{2}\right\rangle$ shown in figure 3, both for $\alpha \equiv u$ (figure $3 a$ ) and $\alpha=v$ (figure $3 b$ ), are inferred from temporal autocorrelations via Taylor's hypothesis. Clearly, the organization can be more readily distinguishable in $\rho_{v}$. In particular, the $\rho_{v}$ distributions for the screen strip and screen cylinder exhibit significant quasiperiodicity, which is consistent with previous topological information gathered for porous-body wakes (e.g. Zhou \& Antonia 1994, 1995). Zhou \& Antonia (1994) noted that at $x / d=20$, the Strouhal number corresponding to the peak in the $v$ spectrum was significantly larger $(\simeq 0.28)$ for the screen-strip wake than for circular and triangular cylinders $(\simeq 0.20)$ and a square cylinder $(\simeq 0.12)$. Although the vortex street disappears when the solidity of the screen is less than about $80 \%$ (Castro 1971), the near-wake region is dominated (Huang \& Keffer 1996) by the vortices in the mixing layers originating from the edges of the mesh. The sectional streamlines obtained by Zhou \& Antonia revealed the presence of mixing-layer vortices while recent flow visualizations, carried out using the smoke-wire technique, have confirmed that these vortices undergo pairing thus supporting the suggestion (Antonia \& Mi 1998) that the strength of these vortices increases with $x$ in the near wake. No visualizations have been made for the screen cylinder wake; it is unlikely that this flow will be dominated 


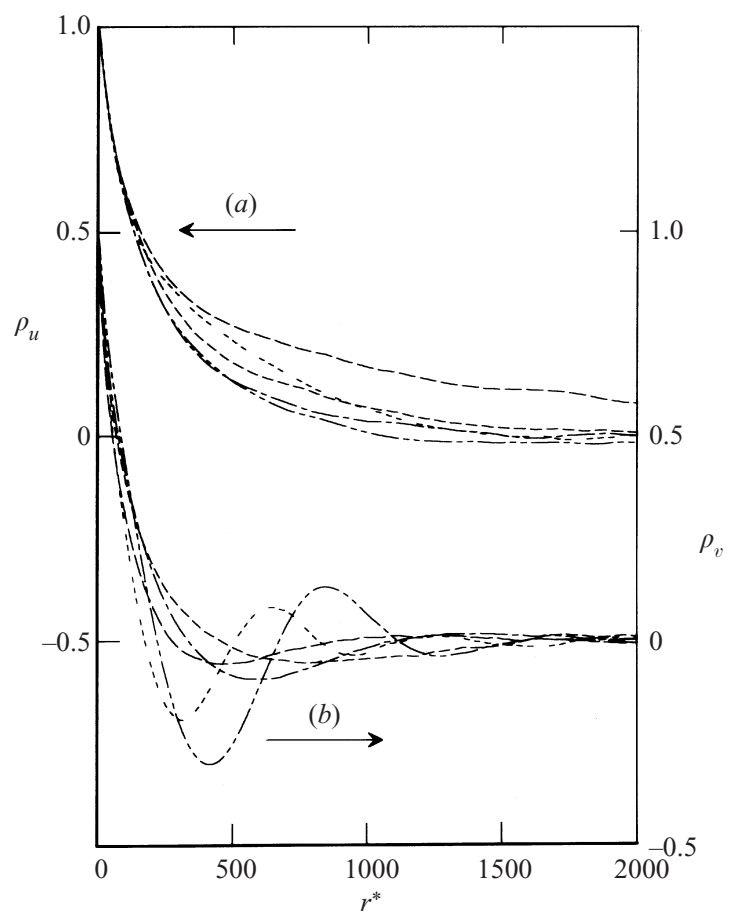

FIGURE 3. Autocorrelation coefficients of $u$ and $v$ in each wake at $R_{\lambda} \simeq 200 .(a) \rho_{u} ;(b) \rho_{v}$. ---, solid circular cylinder; - - - - - solid square cylinder; $-\ldots$, solid normal plate; $-\ldots$, screen strip; - - , circular screen cylinder.

by the evolution of mixing layers that is a feature of the screen strip wake. Although figure 3 points to a strong quasi-periodicity for the screen cylinder wake, it is plausible that the flow topology associated with this generator will differ substantially from that corresponding to the screen strip. This issue needs to be investigated further.

Estimates of the integral length scale $L_{\alpha}(\alpha \equiv u$ or $v)$ were obtained by integrating $\rho_{\alpha}$, namely $L_{\alpha}=U \int_{0}^{r_{\alpha}} \rho_{\alpha}(r) \mathrm{d} r$, where $r_{\alpha}$ denotes the separation at which $\rho_{\alpha}$ is zero and stops oscillating $\left(r_{\alpha}\right.$ corresponds essentially to the first zero crossing for $\alpha \equiv u$ but not for $\alpha \equiv v$ ). The ratio $L_{v} / L_{u}$ is plotted against $R_{\lambda}$ in figure 4 . As for $\left\langle v^{2}\right\rangle /\left\langle u^{2}\right\rangle$, the magnitude of $L_{v} / L_{u}$ varies appreciably between the different wakes, due principally to changes in $L_{v}$. There is however no perfect correlation between the two ratios although they both are smallest in the solid cylinder wake. $L_{v} / L_{u}$ is largest for the normal plate whereas the largest value of $\left\langle v^{2}\right\rangle /\left\langle u^{2}\right\rangle$ is obtained in the screen strip wake. The ratio $L_{v} / L_{u}$ also provides a measure of isotropy since $\int_{0}^{\infty} \rho_{v}(r) \mathrm{d} r=(1 / 2) \int_{0}^{\infty} \rho_{u}(r) \mathrm{d} r$ when isotropy is assumed. Figure 4 suggests that in all cases $L_{v} / L_{u}$ is significantly smaller than the isotropic value of 0.5 .

Perhaps paradoxically, indirect information about the influence of the large scales on the small scales may be inferred by simply considering the value (appropriately normalized) of the mean energy dissipation rate $\langle\epsilon\rangle$. Indeed, $\langle\epsilon\rangle$ is expected to become independent of $v$ when the Reynolds number is sufficiently large. There is significant evidence to indicate that while the dimensionless parameter $C_{\epsilon} \equiv\langle\epsilon\rangle L_{u} / u^{\prime 3}$ asymptotes to a constant value at sufficiently large $R_{\lambda}$, its magnitude depends on the nature of the flow and, in a given flow type, on the initial flow conditions. In view of the range of initial conditions covered in the present study, it is of interest to examine 


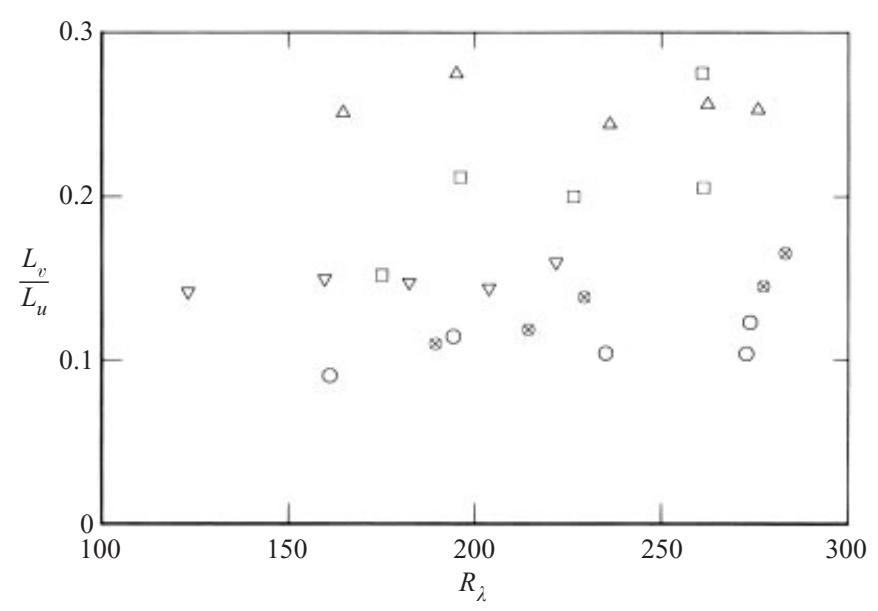

FIGURE 4. Variation with $R_{\lambda}$ of the integral length scale ratio $L_{v} / L_{u}$ in each of the wakes. Symbols are as in figure 2.

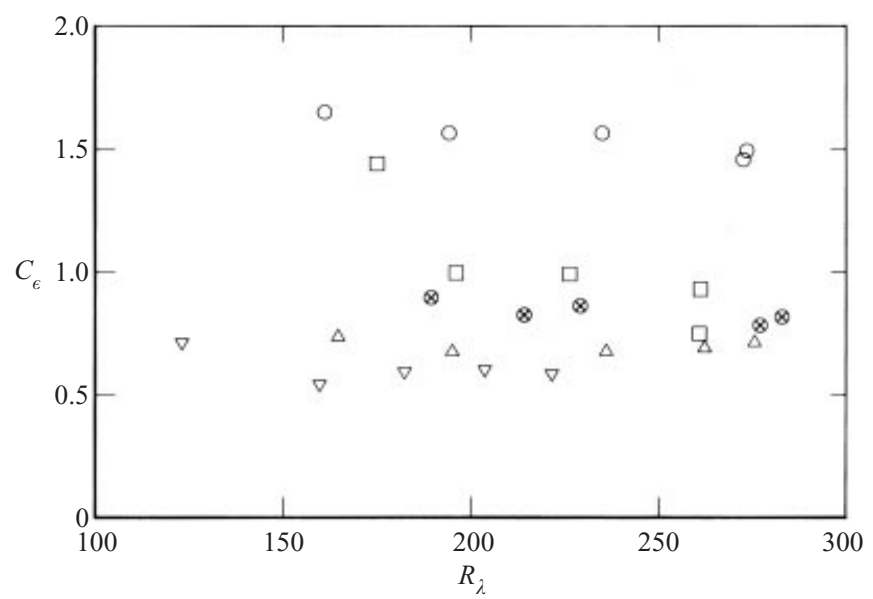

FIGURE 5. Variation with $R_{\lambda}$ of the mean energy dissipation rate parameter $C_{\epsilon}\left(\equiv\langle\epsilon\rangle L_{u} / u^{\prime 3}\right)$ in each of the wakes. Symbols are as in figure 2.

the behaviour of $C_{\epsilon}$. Figure 5 shows that indeed $C_{\epsilon}$ depends significantly on the initial conditions. Its magnitude is as small as 0.6 for the screen strip wake and as large as 1.6 in the wake of the solid cylinder. Comparison between figures 2 and 5 indicates that $C_{\epsilon}$ is small when $\left\langle v^{2}\right\rangle /\left\langle u^{2}\right\rangle$ is large and vice versa; this implies a connection between $\langle\epsilon\rangle$, a small-scale quantity, and the anisotropy of the large-scale motion. Values of $C_{\epsilon}$ were recently reported by Antonia \& Pearson (2000) for the solid cylinder and flat plate wakes; the measurements were made in the same tunnel but $d$ was slightly bigger $(28.25 \mathrm{~mm})$ and $x / d(\simeq 54)$ smaller. Whilst the values of $C_{\epsilon}$ agree closely for the flat plate, the values of $C_{\epsilon}$ in the solid cylinder wake were larger ( $C_{\epsilon}$ decreased from about 2 at $R_{\lambda}=200$ to about 1.8 at $R_{\lambda} \simeq 300$ ), possibly implying a greater sensitivity to initial conditions for the circular cylinder wake than for wakes (e.g. from the flat plate) where the separation points are fixed.

Isotropic estimates of $\langle\epsilon\rangle$ were used for figure 5. Although there are departures from isotropy at the level of the derivative variances contained in $\langle\epsilon\rangle$, the deviation tends to be in opposite directions for the different derivatives we were able to measure; this 


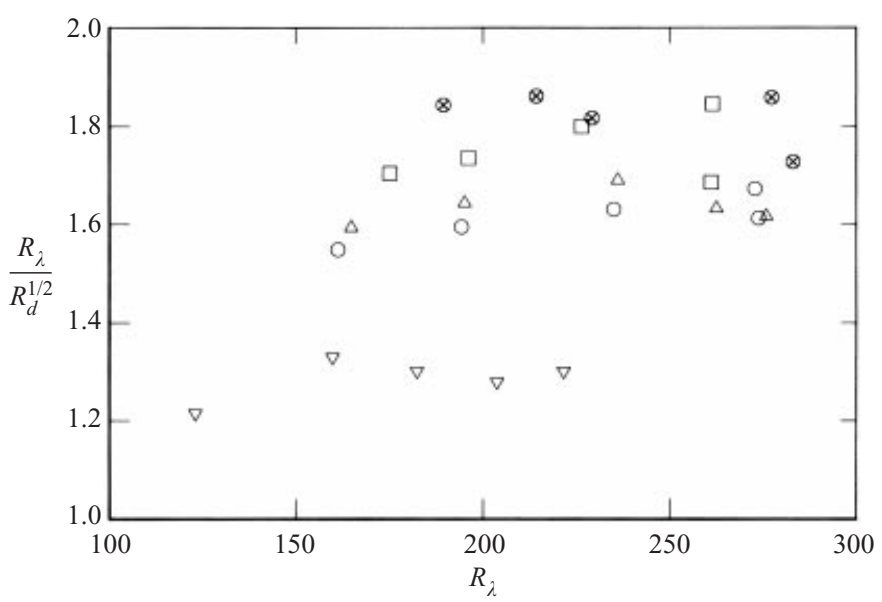

FIGURE 6. Dependence of the Taylor microscale Reynolds number $R_{\lambda}$ on $R_{d}^{1 / 2}$, where $R_{d}$ is the Reynolds number based on the free-stream velocity and characteristic dimension of the wake generator. Symbols are as in figure 2.

may have a compensating effect in terms of producing a near equality between the true value of $\langle\epsilon\rangle$ and its isotropic counterpart $\langle\epsilon\rangle_{\text {iso }}$ so that the effect on figure 5 is unlikely to be significant. Information presented in $\S 7$ suggests that the departures from isotropy are similar in each wake; this feature indicates that the connection between $C_{\epsilon}$ and $\left\langle v^{2}\right\rangle /\left\langle u^{2}\right\rangle$ shown in figure 5 is not affected by the assumption of local isotropy used in determining $\langle\epsilon\rangle$. Further, the use of $\left\langle\omega_{z}^{2}\right\rangle$ data via the (isotropic) relation $\langle\epsilon\rangle=3 v\left\langle\omega_{z}^{2}\right\rangle$ yielded values of $C_{\epsilon}$ which are essentially identical to those in figure 5 (to avoid crowding the figure, values of $C_{\epsilon}$ estimated using $\langle\epsilon\rangle=3 v\left\langle\omega_{z}^{2}\right\rangle$ are not shown in figure 5).

It is finally worth commenting on how the local value of $R_{\lambda}$ is related to $R_{d}$, i.e. the Reynolds number that is generally quoted in wake studies. For a self-preserving wake, it can be shown, starting with the definition of $\lambda$ and the use of isotropy $\left[\langle\epsilon\rangle=15 v\left\langle(\partial u / \partial x)^{2}\right\rangle\right]$, that on the flow centreline

$$
R_{\lambda}=15^{1 / 2} C_{\epsilon}^{-1 / 2} C_{1}^{2} C_{2}^{1 / 2} C_{3}^{1 / 2} R_{d}^{1 / 2},
$$

with $u^{\prime} / U_{0}=C_{1}, U_{0} / U_{1}=C_{2}\left[\left(x-x_{0}\right) / d\right]^{-1 / 2}$ and $L / d=C_{3}\left[\left(x-x_{0}\right) / d\right]^{1 / 2}(L$ is the mean velocity half-width, $x_{0}$ is the effective flow origin, $U_{0}$ is the maximum velocity defect and $U_{1}$ is the free-stream velocity). Figure 6 indicates that the ratio $R_{\lambda} / R_{d}^{1 / 2}$ is approximately constant in each wake, as indicated by (3.1). The magnitude of the ratio varies significantly from flow to flow, which is not inconsistent with (3.1) since $C_{\epsilon}, C_{1}, C_{2}, C_{3}$ may all depend on the initial conditions. Note that the trend exhibited in figure 6 is not easily reconcilable with that in figure 5. For example, the smallest values of $R_{\lambda} / R_{d}^{1 / 2}$ occur in the wake from the screen strip whereas $C_{\epsilon}$ is smallest in this flow. It is possible that the effect due to $C_{\epsilon}$ is more than cancelled by that from the other three parameters. We do not have data for $C_{2}$ and $C_{3}$ and cannot therefore comment on whether the present data are fully consistent with (3.1).

It should be underlined however that the assumptions (self-preservation and local isotropy) used in deriving (3.1) are, at best, only approximately satisfied by the present experiments. Nonetheless, the results of figure 6 serve as a reminder that, in the context of designing an experiment with the aim of achieving a particular value or range of values for $R_{\lambda}$, the effect of initial conditions cannot be ignored. 


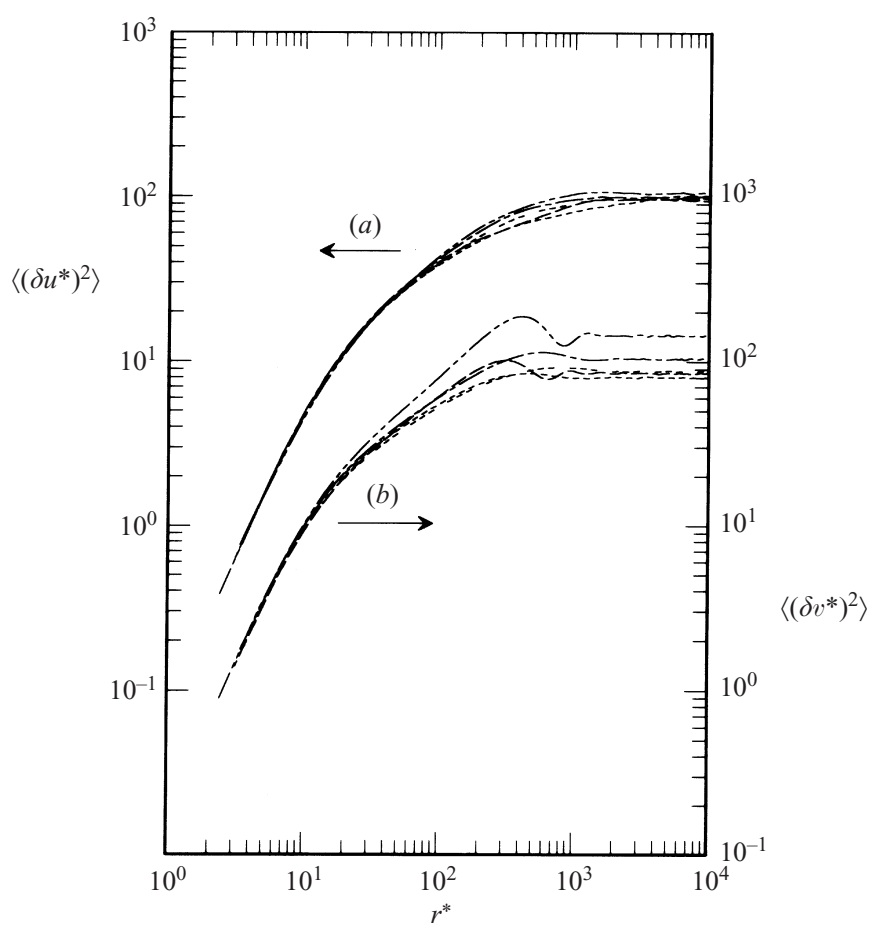

FIGURE 7. Kolmogorov-normalized second-order velocity structure functions in each wake $\left(R_{\lambda} \simeq 200\right)$. Line types are as in figure 3. $(a)\left\langle\left(\delta u^{*}\right)^{2}\right\rangle ;(b)\left\langle\left(\delta v^{*}\right)^{2}\right\rangle$.

\section{Velocity structure functions}

In view of the recent evidence regarding the dependence on $R_{\lambda}$ of Kolmogorov normalized second and higher even order velocity structure functions over the dissipative and inertial ranges of scales (Pearson 1999; Antonia et al. 2000), it is important to compare $\left\langle\left(\delta u^{*}\right)^{2}\right\rangle$ and $\left\langle\left(\delta v^{*}\right)^{2}\right\rangle$, obtained in the different wakes at approximately the same value of $R_{\lambda}$. Distributions of $\left\langle\left(\delta u^{*}\right)^{2}\right\rangle$ and $\left\langle\left(\delta v^{*}\right)^{2}\right\rangle$ are plotted in figures $7(a)$ and $7(b)$ against $r^{*}$. For values of $r^{*}$ in the dissipative range (roughly $r^{*} \leqslant 10$ ) there is reasonable collapse for $\left\langle\left(\delta u^{*}\right)^{2}\right\rangle$ whereas the collapse for $\left\langle\left(\delta v^{*}\right)^{2}\right\rangle$ is of poorer quality. This behaviour is not surprising since

$$
\underset{r^{*} \rightarrow 0}{\mathscr{L} t}\left\langle\left(\delta u^{*}\right)^{2}\right\rangle=\frac{r^{* 2}}{15}
$$

when local isotropy is assumed. Since $\langle\epsilon\rangle_{\text {iso }}$ was used here, the collapse for $\left\langle\left(\delta u^{*}\right)^{2}\right\rangle$ is essentially ensured. On the other hand (the assumption of local isotropy is retained)

$$
\underset{r^{*} \rightarrow 0}{\mathscr{L} t}\left\langle\left(\delta v^{*}\right)^{2}\right\rangle=a \frac{r^{* 2}}{15}
$$

with $a \equiv\left\langle(\partial v / \partial x)^{2}\right\rangle /\left\langle(\partial u / \partial x)^{2}\right\rangle$. The lack of collapse in $\left\langle\left(\delta v^{*}\right)^{2}\right\rangle$ (figure $\left.7 b\right)$ reflects slight departures from local isotropy, namely slight departures of $a$ from 2, its isotropic value. At the largest values of $r^{*}$ (in excess of $L_{u}^{*}$ or $L_{v}^{*}$ ), there is nearly perfect collapse for $\left\langle\left(\delta u^{*}\right)^{2}\right\rangle$ but the distributions for $\left\langle\left(\delta v^{*}\right)^{2}\right\rangle$ vary appreciably. This behaviour is consistent with the following expectations (local isotropy is once more 
assumed):

$$
\underset{r^{2} \rightarrow \infty}{\mathscr{L} t}\left\langle\left(\delta u^{*}\right)^{2}\right\rangle=2\left\langle u^{* 2}\right\rangle=2(15)^{-1 / 2} R_{\lambda}
$$

and

$$
\underset{r^{*} \rightarrow \infty}{\mathscr{L} t}\left\langle\left(\delta v^{*}\right)^{2}\right\rangle=2\left\langle v^{* 2}\right\rangle=2\left(\frac{\left\langle v^{2}\right\rangle}{\left\langle u^{2}\right\rangle}\right)(15)^{-1 / 2} R_{\lambda} .
$$

Since $R_{\lambda}$ is constant, $\left\langle\left(\delta u^{*}\right)^{2}\right\rangle$ must remain constant. The level of support for this by the results in figure $7(a)$ underlines that departures from local isotropy, an assumption used in estimating $\langle\epsilon\rangle$, are small. Accordingly, the large variation in the limiting value (large $\left.r^{*}\right)$ of $\left\langle\left(\delta v^{*}\right)^{2}\right\rangle$ exhibited in figure $7(b)$ cannot be attributed to departures from local isotropy. The large- $r^{*}$ limit of $\left\langle\left(\delta v^{*}\right)^{2}\right\rangle$ is expected to be constant (for different wakes) provided $\left\langle v^{2}\right\rangle /\left\langle u^{2}\right\rangle$ is constant. Isotropy at all scales (or global isotropy) requires $\left\langle v^{2}\right\rangle /\left\langle u^{2}\right\rangle$ to be 1 . The results of figure 2 indicate that this requirement is clearly violated in the present experiments.

Although the $\left\langle\left(\delta u^{*}\right)^{2}\right\rangle$ distributions approach a plateau at large $r^{*}$ in a relatively smooth fashion, most of the $\left\langle\left(\delta v^{*}\right)^{2}\right\rangle$ distributions exhibit oscillations before becoming constant. This behaviour is most noticeable for the two porous-body wakes, the largest oscillation occurring in the wake from the screen strip. The strong oscillations of $\left\langle\left(\delta v^{*}\right)^{2}\right\rangle$ for the porous-body wakes are consistent with the topological features observed by Zhou \& Antonia $(1994,1995)$ for such types of wakes and with the present autocorrelations of $v$ in figure $3(b)$.

It almost follows from the behaviour of $\left\langle\left(\delta v^{*}\right)^{2}\right\rangle$ at large $r^{*}$ that the difference in the large-scale motion between the various wakes will be felt at smaller vales of $r^{*}$, almost certainly those that would normally be associated with the inertial range and arguably those that are identifiable with the dissipative range. The imperfect collapse in the range $r^{*} \leqslant 10$ very likely reflects, at least for the present moderate value of $R_{\lambda}$, the influence of the anisotropic large-scale motion on the isotropy of the smallest scales. The effect on scales in the range $20 \leqslant r^{*} \leqslant 200$ is significant, especially for $\left\langle\left(\delta v^{*}\right)^{2}\right\rangle$. That this effect is inextricably linked to the difference between the large-scale motions in the different wakes is strongly supported by the fact that other parameters, such as $R_{\lambda}$ and the local mean shear, which can affect this range of scales, have been kept constant. It was of interest here to examine $\left\langle\left(\delta u^{*}\right)^{2}\right\rangle$ and $\left\langle\left(\delta v^{*}\right)^{2}\right\rangle$ obtained in the same wake but over a range of $R_{\lambda}$. Distributions of $\left\langle\left(\delta v^{*}\right)^{2}\right\rangle$ are shown in figure 8 for only two wakes over the range of $R_{\lambda}$ covered in the present experiments. In the range $20 \leqslant r^{*} \leqslant 200$, the variation of $\left\langle\left(\delta v^{*}\right)^{2}\right\rangle$ in the solid body wake (figure $8 a$ ) is comparable to the variations (figure $7 b)$ of $\left\langle\left(\delta v^{*}\right)^{2}\right\rangle$ between different wakes (at $R_{\lambda}=200$ ). For the porous-body wake (figure $8 b$ ) the variation with respect to $R_{\lambda}$ is smaller than that indicated in figure $7(b)$.

The variation in local slope, over the range $20 \leqslant r^{*} \leqslant 200$, of the distributions of $\left\langle\left(\delta u^{*}\right)^{2}\right\rangle$ and $\left\langle\left(\delta v^{*}\right)^{2}\right\rangle$ in figures $7(a)$ and $7(b)$ implies that the concept of an inertial range (over which $\left\langle\left(\delta \alpha^{*}\right)^{2}\right\rangle$ has a power-law behaviour) may not be tenable here. To highlight this, the local slope $\beta_{\alpha} \equiv \mathrm{d}\left[\log _{10}\left\langle\left(\delta \alpha^{*}\right)^{2}\right\rangle\right] / \mathrm{d}\left(\log _{10} r^{*}\right)$ is plotted in figure 9 . The magnitude of $\beta_{\alpha}$ must vary between 2 at small $r^{*}$, since $\left\langle\left(\delta \alpha^{*}\right)^{2}\right\rangle \sim r^{* 2}$ as $r^{*} \rightarrow 0$, and 0 at large $r^{*}$ since $\left\langle\left(\delta \alpha^{*}\right)^{2}\right\rangle \rightarrow 2\left\langle\alpha^{* 2}\right\rangle$ as $r^{*} \rightarrow \infty$. Following the sharp decrease across the dissipative range, the magnitude of $\beta_{\alpha}$ decreases more slowly over the range $20 \leqslant r^{*} \leqslant 200\left(1.3 \leqslant \log _{10} r^{*} \leqslant 2.3\right)$; somewhat surprisingly, this is more discernible in $\left\langle\left(\delta v^{*}\right)^{2}\right\rangle$ (figure $\left.9 b\right)$ than $\left\langle\left(\delta u^{*}\right)^{2}\right\rangle$ (figure $9 a$ ). There is however no region over which $\beta_{\alpha}$ can be regarded as constant, thus emphasizing that there is no range over which 


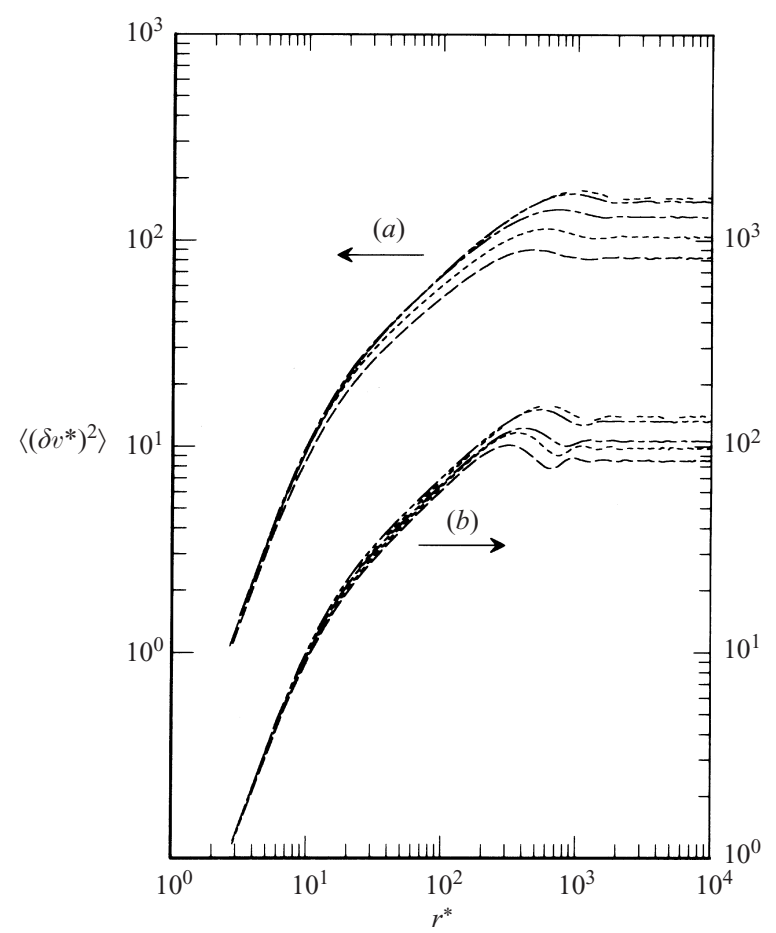

FiguRE 8. Dependence on $R_{\lambda}$ of Kolmogorov-normalized second-order moments of $\delta v$ in two wake flows. (a) Solid flat plate: -,$R_{\lambda}=165 ;--, 195 ;-\ldots, 236 ;-\ldots$, , 262; - - - - 276. (b) Circular screen cylinder: $R_{\lambda}=189 ;--, 214 ; \ldots-\ldots, 229 ;-\ldots-, 277 ;-\ldots-., 283$.

$\left\langle(\delta \alpha)^{2}\right\rangle$ has a power-law dependence and implying that the inertial range concept of $\mathrm{K} 41$ is not valid in the present context. For reference, the K41 value of $\beta_{\alpha}(=2 / 3)$ is shown in figures $9(a)$ and $9(b)$.

Further confirmation of lack of homogeneity and isotropy in the range $20 \leqslant r^{*} \leqslant$ 200 is provided by figure 10 where $-\left\langle\left(\delta u^{*}\right)^{3}\right\rangle / r^{*}$ is shown for each wake. Despite $R_{\lambda}$ remaining constant, there is an appreciable variation in the distributions. None of the distributions suggest the existence of a region where $-\left\langle\left(\delta u^{*}\right)^{3}\right\rangle$ varies linearly with $r^{*}$. In particular, the peak value of $-\left\langle\left(\delta u^{*}\right)^{3}\right\rangle / r^{*}$ is significantly smaller than the value of $4 / 5$ predicted by Kolmogorov $(1941 b)$ for locally homogeneous and isotropic turbulence. Its magnitude and the corresponding location vary significantly between different wakes. The overall trend in figure 10 points to a different kind of inhomogeneity in almost every wake. This inhomogeneity would need to be retained in the Navier-Stokes equations in order to 'generalize' the Kolmogorov equation; such an approach was recently adopted by Danaila et al. $(1999,2001)$ both for grid turbulence and the central region of a fully developed turbulent channel flow. Although this approach has not been applied to the present flow, it should be noted that, in the limit of large $r^{*}$, the 'generalized' equation would need to correctly reflect the turbulent energy budget on the wake centreline, i.e. that $\langle\epsilon\rangle$ is balanced by the turbulent advection and the turbulent diffusion. Further, the relative magnitudes of these three quantities are likely to vary slightly among the different wakes.

Isotropy requires that $\left\langle\left(\delta v^{*}\right)^{3}\right\rangle$ is zero everywhere. Figure 11 indicates that whilst, arguably, this requirement is satisfied for $r^{*} \leqslant 10,\left\langle\left(\delta v^{*}\right)^{3}\right\rangle$ varies appreciably in the region $20 \leqslant r^{*} \leqslant 200$. Note that the scale used for the ordinate in figure 11 


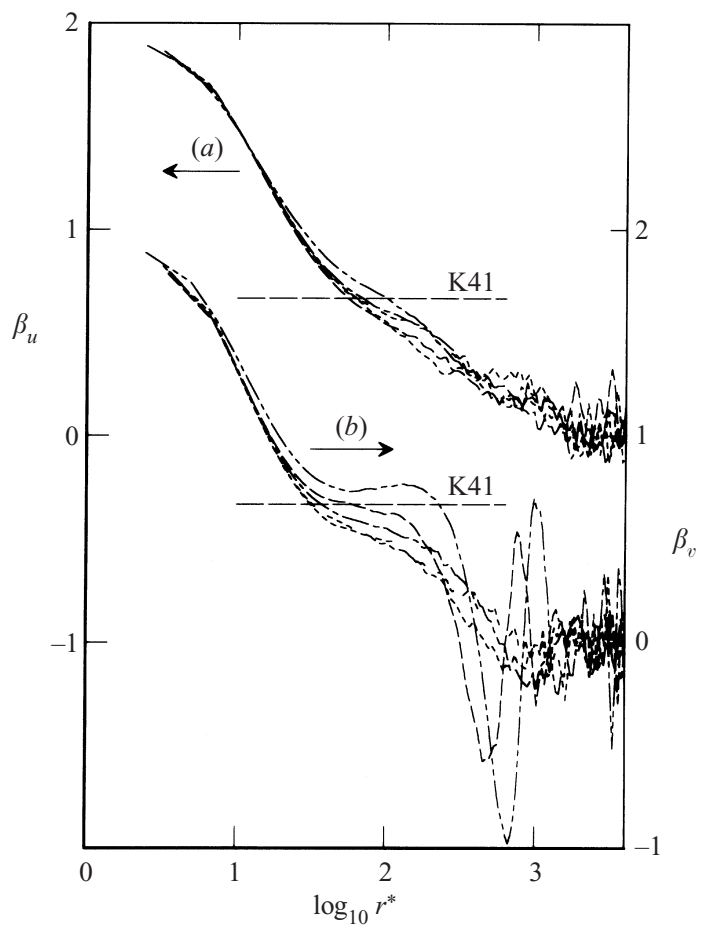

FIGURE 9. Distributions for each wake of $\beta_{\alpha}$, the derivative with respect to $\log _{10} r^{*}$ of $\log _{10}\left\langle\left(\delta \alpha^{*}\right)^{2}\right\rangle$. $R_{\lambda} \simeq 200$. Line types are as in figure 3. (a) $\beta_{u} ;(b) \beta_{v}$.

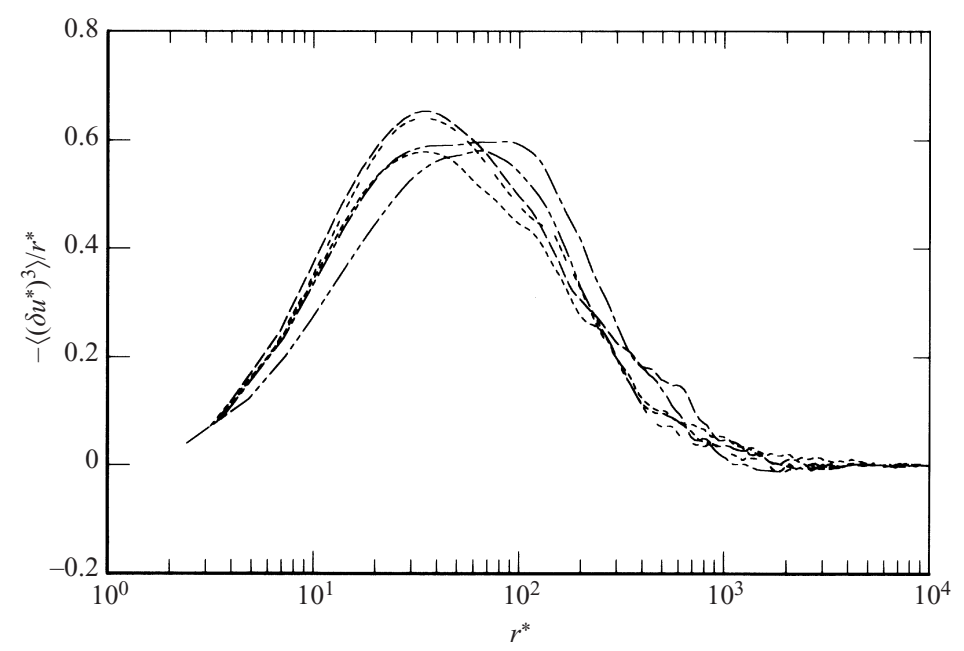

FIGURE 10. Distributions of $-\left\langle\left(\delta u^{*}\right)^{3}\right\rangle / r^{*}$ in each wake at $R_{\lambda} \simeq 200$. Line types are as in figure 3 .

is considerably bigger than that in figure 10 in order to accommodate the large excursions of $\left\langle\left(\delta v^{*}\right)^{3}\right\rangle$ in the screen strip wake. For the other wakes, the variation cannot be neglected, either in terms of its magnitude or its sign (for $r^{*} \gtrsim 10,\left\langle\left(\delta v^{*}\right)^{3}\right\rangle$ can either increase or decrease with respect to $r^{*}$ ). 


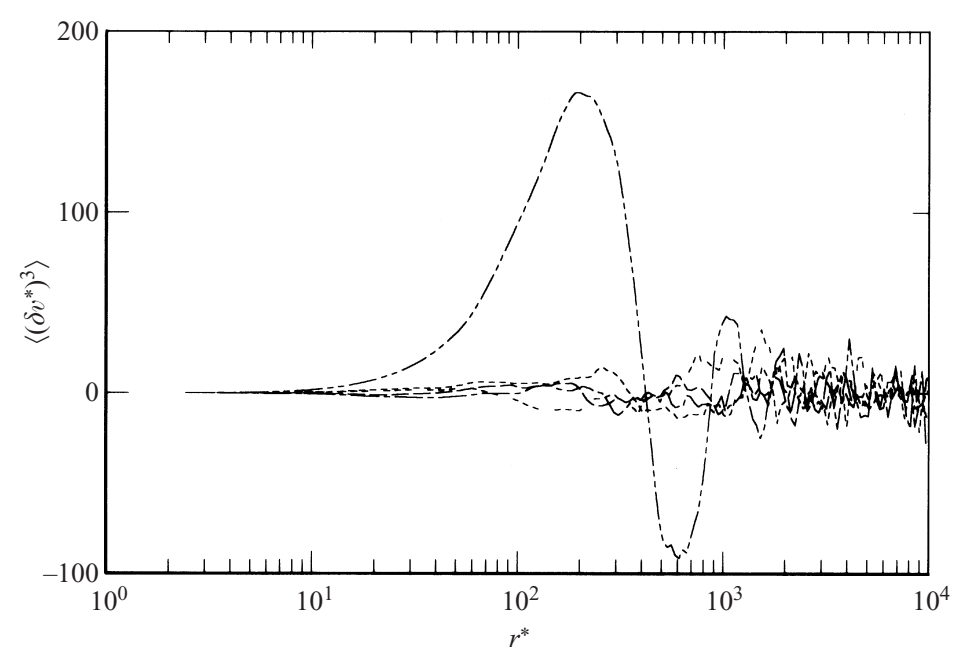

FIGURE 11. Distributions of $\left\langle\left(\delta v^{*}\right)^{3}\right\rangle$ in each wake at $R_{\lambda} \simeq 200$. Line types are as in figure 3 .

\section{Scaling exponents}

The implication from figure 9 is that it is strictly not possible to quote unique values of the scaling exponents associated with the second-order velocity structure functions. It may be possible to 'quote' values of $\beta_{\alpha}$ which represent averages over the scaling range. This was not attempted as it involves some arbitrariness in selecting the beginning and end of the range. We have however adopted the widespread practice of estimating 'relative' values of the exponents via the extended self-similarity (ESS) method (Benzi et al. 1993). In this method, the distributions of $\left\langle\left|(\delta \alpha)^{n}\right|\right\rangle$ are plotted against $\left\langle\left|(\delta u)^{3}\right|\right\rangle$ with the slopes usually being determined over a range for which $\left\langle|\delta u|^{3}\right\rangle$ is assumed to increase linearly with $r^{*}$. In reality, like $\left\langle\left(\delta u^{*}\right)^{3}\right\rangle,\left\langle\left|\left(\delta u^{*}\right)\right|^{3}\right\rangle$ does not increase linearly with $r^{*}$. Figure 12 indicates that the magnitude of the local slope $\mathrm{d}\left[\log _{10}\left\langle\left|\left(\delta u^{*}\right)\right|^{3}\right\rangle\right] / \mathrm{d}\left[\log _{10} r^{*}\right]$ decreases continuously with increasing $r^{*}$, a variation which is qualitatively similar to that exhibited by figure 9. In particular, figure 12 emphasizes that the data do not support a linear behaviour (horizontal line in figure 12).

Notwithstanding the shortcoming of the assumption $\left\langle\left|\left(\delta u^{*}\right)\right|^{3}\right\rangle \sim r^{*}$, it is of interest to compare the ESS values of $\zeta_{\alpha n}$ among the different wakes. Figure 13 shows that the magnitude of $\zeta_{\text {un }}$ does not vary significantly, although the magnitudes for the screen cylinder wake are consistently smaller than for the other wakes, particularly as $n$ increases. Overall, the magnitudes of $\zeta_{u n}$ are in reasonable agreement with those predicted by the log-normal model (Kolmogorov 1962) with an intermittency exponent $\mu$ of 0.2 (e.g. Sreenivasan \& Kailasnath 1993) and the model of She-Leveque (1994); they also agree reasonably well with the measurements of Anselmet et al. (1984) and the simulations of Vincent \& Meneguzzi (1991). As expected, given the moderate value of $R_{\lambda}$, the magnitudes of $\zeta_{v n}$ are, in general, significantly smaller than those of $\zeta_{u n}$. More importantly however, there is a significant variation among the different wakes, in both the magnitude of $\zeta_{v n}$ and also its rate of increase with respect to $n$. For the porous-bodies, the rate decreases as $n$ increases by comparison to that for the solid-body wakes. In figure 14 , the difference $\left(\zeta_{u n}-\zeta_{v n}\right)$ is plotted against $n$. It is evident that, at least for $n \leqslant 6$, this difference is smaller for the porous than the solid-body wakes, the smallest values occurring in the screen strip wake. For this 


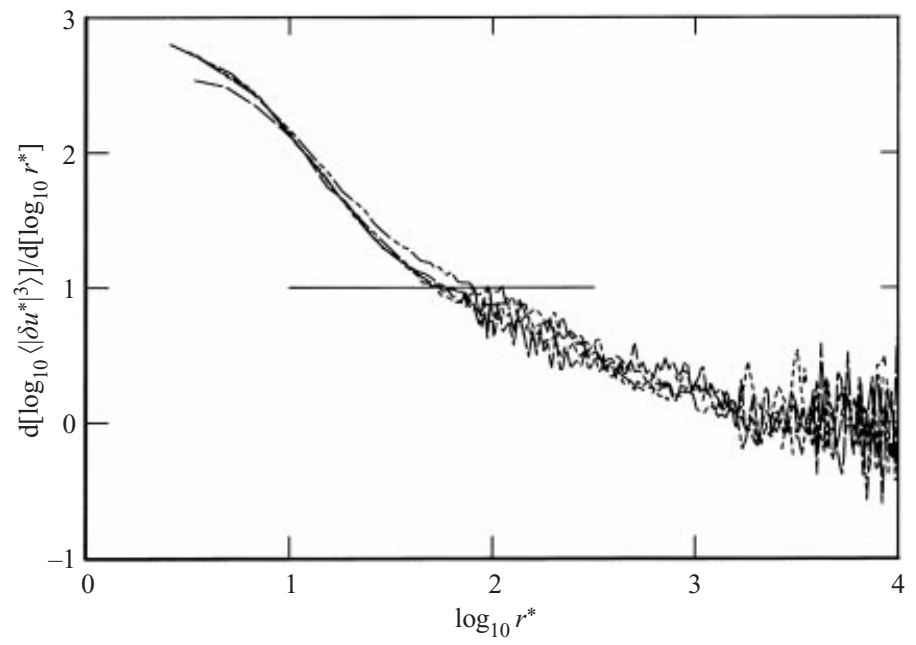

FIGURE 12. Distributions for each wake of the derivative with respect to $\log _{10} r^{*}$ of $\log _{10}\left\langle\left|\left(\delta u^{*}\right)\right|^{3}\right\rangle$ at $R_{\lambda} \simeq 200$. Line types are as in figure 3 .

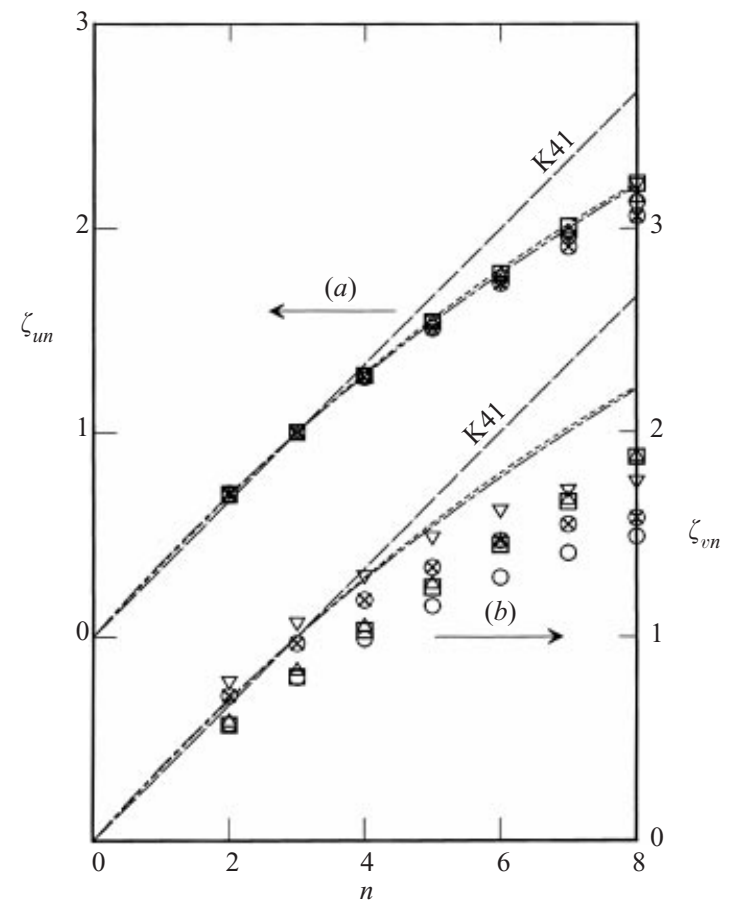

FIGURE 13. ESS scaling exponents associated with $\left\langle(\delta u)^{n}\right\rangle$ and $\left\langle(\delta v)^{n}\right\rangle$ as a function of $n$. Note that different origins are used for $\zeta_{n u}$ and $\zeta_{n v}$. Symbols are as in figure $2 .--, \mathrm{K} 41 ;--$, , log-normal model (K62) with $\mu=0.2 ;-\ldots$, model of She-Leveque (1994).

latter flow, $\left(\zeta_{u n}-\zeta_{v n}\right)$ is negative for $n=2,3,4$, apparently reflecting the fact that $\left\langle v^{2}\right\rangle$ is $37 \%$ larger than $\left\langle u^{2}\right\rangle$ in this particular flow (figure 2). The moderately low values of $L_{v} / L_{u}$ for this flow, figure 4 , are a further contributing factor. Figure 15 suggests that there is no clear-cut dependence of $\left(\zeta_{u n}-\zeta_{v n}\right)$ (for $n=2$ and 4$)$ on the ratio $\left\langle v^{2}\right\rangle /\left\langle u^{2}\right\rangle$, due mainly to the disparity in the magnitude of $\left(\zeta_{u n}-\zeta_{v n}\right)$ between the screen cylinder 


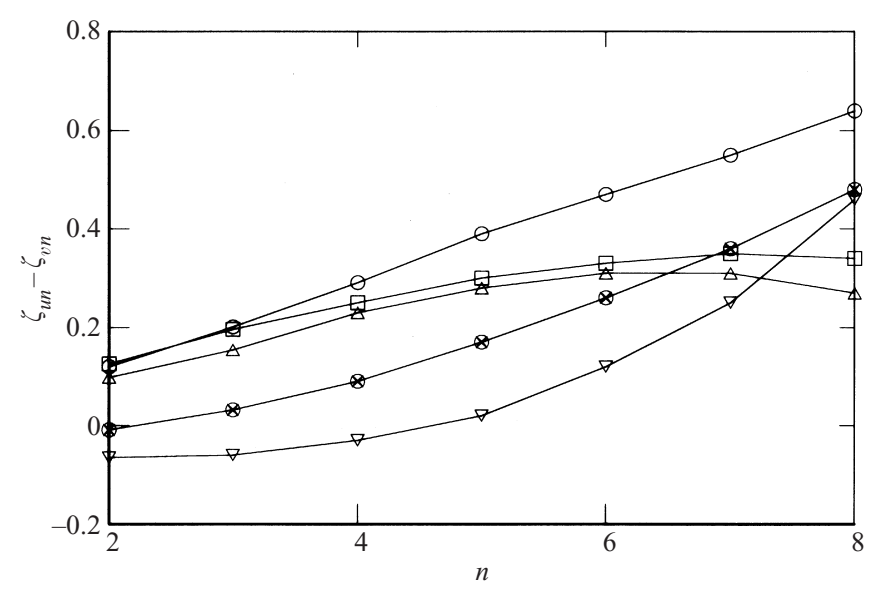

FIGURE 14. Dependence of the difference $\left(\zeta_{u n}-\zeta_{v n}\right)$ between longitudinal and transverse ESS scaling exponents on $n$. Results are shown for each wake $\left(R_{\lambda} \simeq 200\right)$. Symbols are as in figure 2 .

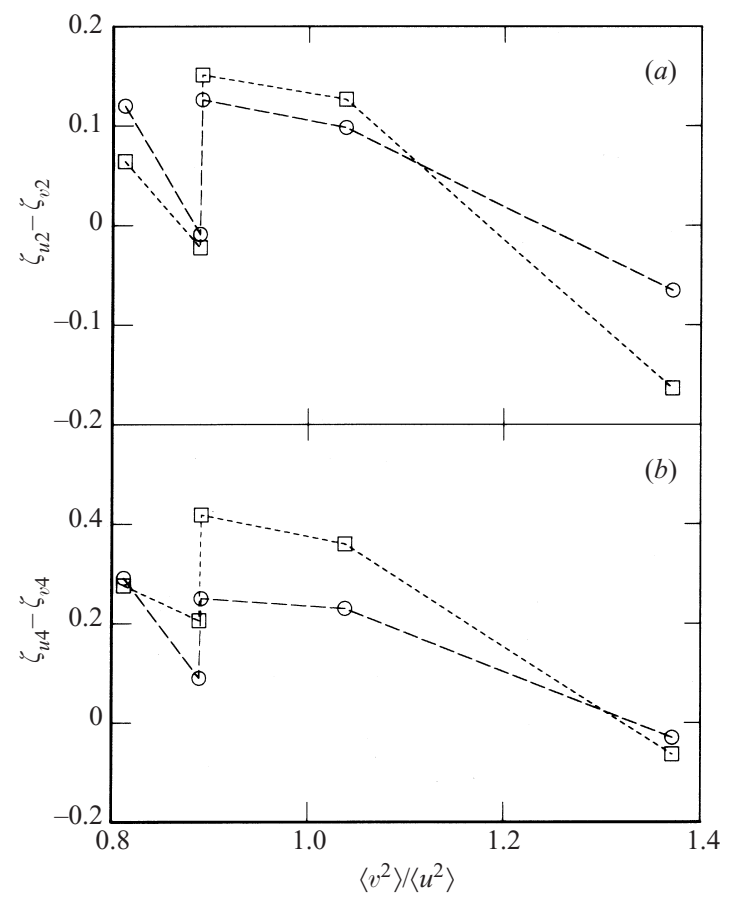

FIGURE 15. Dependence of the difference $\left(\zeta_{u n}-\zeta_{v n}\right)$, for $n=2$ and 4 , between longitudinal and transverse ESS scaling exponents on $\left\langle v^{2}\right\rangle /\left\langle u^{2}\right\rangle$. Results are shown for each wake $\left(R_{\lambda} \simeq 200\right)$. (a) $n=2$; (b) $n=4$ : $\bigcirc---\bigcirc$, measured; $\square---\square$, estimates using model (equation (5.1)).

wake and the square cylinder wake (for these two flows, $\left\langle v^{2}\right\rangle /\left\langle u^{2}\right\rangle$ is approximately the same, equal to about 0.89$)$. Nevertheless, the lower values of $\left(\zeta_{u n}-\zeta_{v n}\right)$ for the screen cylinder wake are commensurate with the smaller value of $L_{v} / L_{u}$ in this flow, relative to the square cylinder wake. Further, they can be captured by a model (Romano \& Antonia 2001) described briefly below, which takes into account the effect of both $\left\langle v^{2}\right\rangle /\left\langle u^{2}\right\rangle$ and $L_{v} / L_{u}$ on the relative behaviours of $\left\langle\left(\delta u^{*}\right)^{2}\right\rangle$ and $\left\langle\left(\delta v^{*}\right)^{2}\right\rangle$.

The main requirement of the model is that the asymptotic behaviour of $n$ th-order 
structure functions (e.g. Frisch 1995) at very small and large $r^{*}$ should be reproduced correctly. As $r^{*} \rightarrow 0,\left\langle\left(\delta \alpha^{*}\right)^{n}\right\rangle \rightarrow A_{\alpha}(n) r^{* n}$, whereas as $r^{*} \rightarrow \infty,\left\langle\left(\delta \alpha^{*}\right)^{n}\right\rangle \rightarrow 2 B_{\alpha}(n) \alpha^{\prime^{* n}}$ (where $\alpha \equiv u$ or $v$ and the prefactors $A_{\alpha}(n)$ and $B_{\alpha}(n)$ depend on the Reynolds number). Expressions for $A_{\alpha}(n)$ and $B_{\alpha}(n)$ are given for $n=2,4$ in Romano \& Antonia (2001). It is also necessary to specify the limiting values for $r^{*}$. At the lower end, $r^{*} \sim 1$, whereas $r^{*}$ is taken proportional to $L_{\alpha}^{*}$ at the upper end. Effective length scales (which are multiples of $\eta$ and $L_{\alpha}$ ) are introduced as suggested by Sreenivasan (1995), namely $\eta_{\alpha_{e f f}}^{*}\left(=\eta_{\alpha_{e f f}} / \eta\right)$ and $L_{\alpha_{e f f}}^{*}\left(=L_{\alpha_{e f f}} / \eta\right)$. The scaling exponents of the $n$ th-order structure function are approximated by

$$
\zeta_{\alpha n}=\frac{\log \left[2 B_{\alpha}(n) \alpha^{\alpha^{* n}}\right]-\log \left[A_{\alpha}(n) \eta_{\alpha_{e f f}}^{* n}\right]}{\log \left(L_{\alpha_{e f f}}^{*}\right)-\log \left(\eta_{\alpha_{\text {eff }}}^{*}\right)}=\frac{\log C_{\alpha}(n)}{\log D_{\alpha}}
$$

where $C_{\alpha}(n)=\left(2 B_{\alpha}(n) \alpha^{\prime * n}\right) /\left(A_{\alpha}(n) \eta_{\alpha_{e f f}}^{* n}\right)$ and $D_{\alpha}=L_{\alpha_{e f f}} / \eta_{\alpha_{e f f}}$. The model contains explicit information about $\alpha^{\prime}$ and $L_{\alpha}$, thus allowing estimates of the scaling exponents which take into account the anisotropy of the large scales.

The same values of $\eta_{\alpha_{e f f}}(\simeq 10 \eta), L_{u_{e f f}}\left(\simeq L_{u}\right)$ and $L_{v_{e f f}}\left(\simeq 3 L_{v}\right)$ were chosen for all the wakes. There is some arbitrariness in the choice of $L_{v_{e f f}}$; this choice was mainly dictated by the need to identify the large-scale plateau in $\left\langle\left(\delta v^{*}\right)^{2}\right\rangle$ correctly. The use of $L_{v_{e f f}} \simeq L_{v}$ would have restricted the evaluation of the scaling exponents to the interval $5 \leqslant r^{*} \leqslant 40$, which is likely to be too influenced by dissipative-range scales and, perhaps more importantly, insufficiently affected by large scales. With $L_{v_{\text {eff }}} \simeq 3 L_{v}$, the exponents are evaluated over the range $15 \leqslant r^{*} \leqslant 100$, which is almost identical to that used for $\left\langle(\delta u)^{n}\right\rangle$. The difficulty in choosing $L_{v_{e f f}}$ arises in flows, such as the present wakes, which are characterized by large-scale oscillations in the correlation coefficients (figure 3) and second-order structure functions (figure $7 b$ ) of $v$.

The model-based estimates of $\left(\zeta_{u n}-\zeta_{v n}\right)$ are shown in figure 15 as a function of $\left\langle v^{2}\right\rangle /\left\langle u^{2}\right\rangle$. They are in reasonable agreement with measurements for $n=2$ (figure $15 a$ ). For $n=4$ (figure $15 b$ ), there is some departure from the measured values, especially for the porous wakes. This reflects the inability of the model to mimic the oscillations of $\left\langle(\delta v)^{2}\right\rangle$ in the latter flows. However, both figure 15(a) and figure 15(b) confirm that the overall effect of the different large-scale anisotropies in the different wakes is captured satisfactorily by the model. In particular, the measured differences between the screen and square-cylinder wakes (for which $\left\langle v^{2}\right\rangle /\left\langle u^{2}\right\rangle$ is nearly the same, but $L_{v} / L_{u}$ is different) are also reproduced by the model.

\section{Spectra}

Although structure functions and spectra are mathematically related, the translation between the power-law exponents in the physical and spectral domains is not exact, particularly since the power-law behaviours $\left(r^{\zeta_{\alpha 2}}\right.$ and $k_{1}^{-m_{\alpha}}$, where $\zeta_{\alpha 2}=2 / 3$ and $m_{\alpha}=5 / 3$ with K41) are expected to apply only over finite ranges (e.g. Hou et al. 1998). Antonia \& Smalley (2000) noted that $m_{u}$ is smaller than $\left(1+\zeta_{u 2}\right)$ in a roughwall boundary layer $\left(R_{\lambda} \leqslant 400\right)$. For completeness, Kolmogorov normalized spectra of $u$ and $v$ are presented in figure 16 . The normalized spectral density function $\phi_{\alpha}^{*}\left(k_{1}^{*}\right)$ is defined such that $\int_{0}^{\infty} \phi_{\alpha}^{*}\left(k_{1}^{*}\right) \mathrm{d} k_{1}^{*}=\left\langle\alpha^{* 2}\right\rangle$, where $k_{1}$ is the one-dimensional wavenumber ( $\equiv 2 \pi f / U$ ). Recall that $\left\langle\alpha^{* 2}\right\rangle$ is equal to half the value corresponding to the plateau in $\left\langle\left(\delta \alpha^{*}\right)^{2}\right\rangle$. The expected dependence of $\left\langle\alpha^{* 2}\right\rangle$ on $R_{\lambda}$, e.g. (4.3) and (4.4), is primarily reflected at small wavenumbers since there is seemingly good collapse at large wavenumbers $\left(k_{1}^{*}>0.02\right)$, notwithstanding the contamination due 


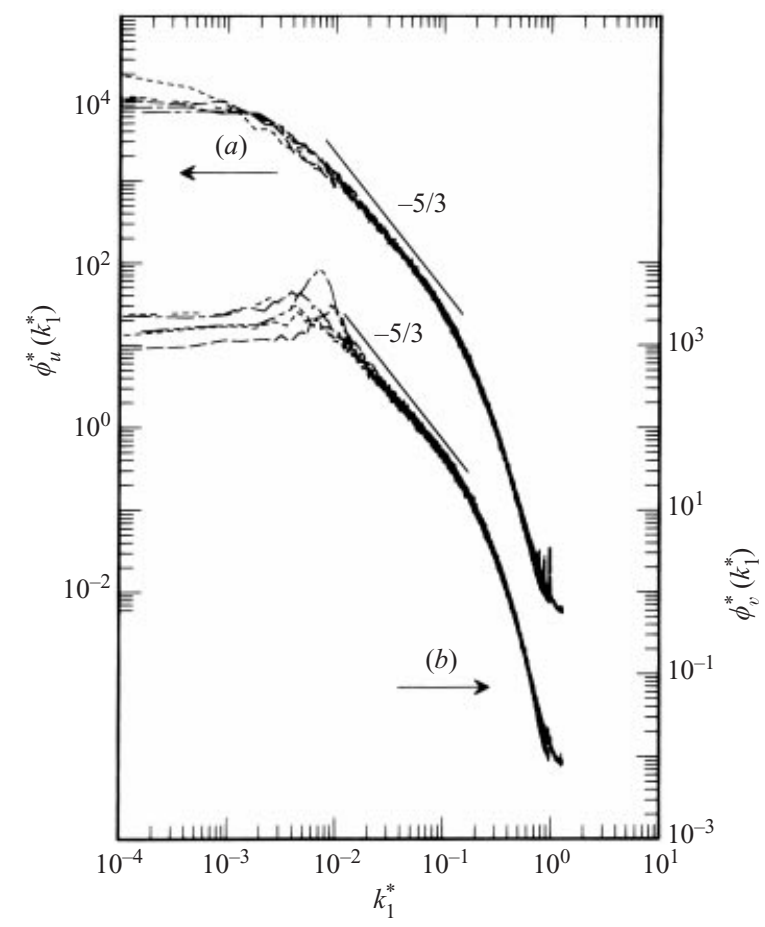

FIGURE 16. Kolmogorov-normalized spectra of $u$ and $v$ at the same $R_{\lambda}(\simeq 200)$ in each of the wakes. Line types are as in figure 3. (a) $u$; (b) $v$.

to electronic noise which is evident near $k_{1}^{*}=1$. The departures among the different wakes, either for $\phi_{u}^{*}\left(k_{1}^{*}\right)$ or $\phi_{v}^{*}\left(k_{1}^{*}\right)$, in the range $k_{1}^{*} \leqslant 0.02$ are not surprising in view of the normalization. However, the use of scales (e.g. $\left\langle\alpha^{2}\right\rangle$ and $L_{\alpha}$ ) associated with the energy-containing structures cannot collapse the curves in this range since the shape and peak values of $\phi_{v}^{*}\left(k_{1}^{*}\right)$ differ, especially betwen porous and solid body wakes. In the context of the spectra, the use of $\langle\epsilon\rangle_{\text {iso }}$ in calculating the Kolmogorov scales imposes the constraint $\int_{0}^{\infty} k_{1}^{* 2} \phi_{\alpha}^{*}\left(k_{1}^{*}\right) \mathrm{d} k_{1}^{*}=$ constant $(1 / 15$ for $\alpha \equiv u$ and $2 / 15$ for $\alpha \equiv v$ ). This is a different type of constraint, possibly a less restrictive one, than that imposed on the structure functions at small $r^{*}$. A possible consequence of the different constraints is the slightly different dependence on $R_{\lambda}$ exhibited by the spectra in figure 17 than that displayed by $\left\langle\left(\delta v^{*}\right)^{2}\right\rangle$ in figure 8 when the focus is on the range of scales lying between the dissipative range and that associated with the large structures. The systematic evolution of $R_{\lambda}$ in figure 8 is much less discernible in figure 17, especially for the screen cylinder (figure 17b). The dependence on $R_{\lambda}$ in the range $k_{1}^{*} \leqslant 0.01$ (figure 17) is as unambiguous as that in figure 8 but it would appear that the plateau in figure 8 exerts a more direct influence on the inertial range than in figure 17. As was noted by Antonia \& Smalley (2000), the power-law behaviour appears (at least on a log-log presentation) more convincing for $\phi_{v}^{*}\left(k_{1}^{*}\right)$ than $\left\langle\left(\delta v^{*}\right)^{2}\right\rangle$. We have not attempted here to estimate $m_{\alpha}$ via a trial and error method by optimizing the 'plateaux' in plots of $k_{1}^{* m_{\alpha}} \phi_{\alpha}^{*}\left(k_{1}^{*}\right)$, as in Mydlarski \& Warhaft (1996), Sreenivasan (1996) or Antonia \& Smalley (2000). However, in order to provide comparison with $\beta_{\alpha}$ (figure 9 ), the velocity spectra were differentiated after first applying a high-order polynomial fit using the procedure of Kim \& Antonia (1993). Distributions of $\gamma_{\alpha} \equiv \mathrm{d}\left[\log _{10} \phi_{\alpha}^{*}\left(k_{1}^{*}\right)\right] / \mathrm{d}\left[\log _{10} k_{1}^{*}\right]$ are shown in figure 18 over 


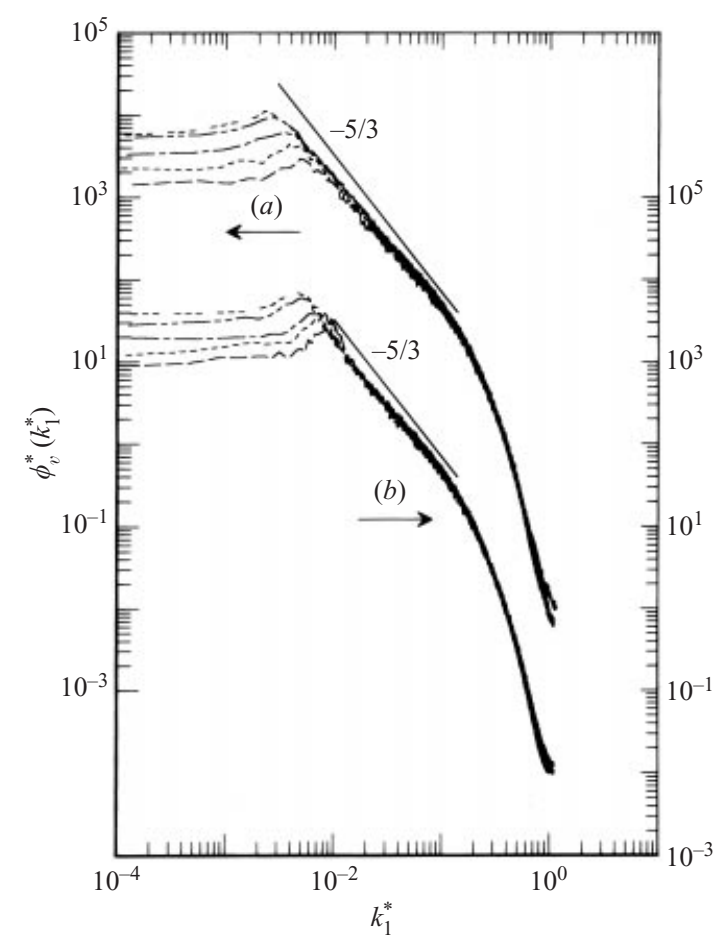

FIGURE 17. Dependence on $R_{\lambda}$ of Kolmogorov-normalized spectra of $v$ in two wake flows: (a) solid flat plate; $(b)$ circular screen cylinder. Line types are as in figure 8.

one decade in wavenumber $\left(0.01 \leqslant k_{1}^{*} \leqslant 0.1\right.$ or $\left.-2 \leqslant \log _{10} k_{1}^{*} \leqslant-1\right)$. In general, there is no region in which $\gamma_{\alpha}$ can be clearly regarded as constant; this is consistent with the earlier inference from figure 9. Figure 18(a) indicates that, except for the screen strip wake, $\gamma_{u}$ continually decreases as $k_{1}^{*}$ increases, with a possible tendency towards a plateau for the square cylinder and flat-plate wakes. By contrast, all the $\gamma_{v}$ distributions (figure 18b) exhibit a waviness; this is more pronounced for the porousbody wakes. If an average value of $\left(-\gamma_{\alpha}\right)$ is loosely identified with $m_{\alpha}$, the magnitude of this latter quantity is larger for the porous-body wakes than the solid-body wakes at least when $\alpha \equiv v$. In the porous wakes, $m_{v}$ is closer to the K41 value $(5 / 3)$ than for the other three wakes. The largest values of $m_{u}$ and $m_{v}$ are observed for the screen strip; this is consistent with the indication from figure 9 that the largest values of $\beta_{\alpha}$ also occur for this flow.

\section{Vorticity characteristics}

It follows from its definition that vorticity fluctuations should be more characteristic of the small-scale motion than the velocity fluctuations. It is therefore of interest to consider various statistics of $\omega_{z}$, such as its variance, p.d.f. and spectral density functions. Because of the imperfect spatial resolution of the probe, the high-wavenumber part of the vorticity spectrum is attenuated. Corrections for this attenuation were made, by assuming local isotropy, according to the procedures outlined in Antonia, Shafi \& Zhu (1996). Distributions of the corrected spectra $\phi_{\omega_{z}}^{*}\left(k_{1}^{*}\right)$ are shown in figure 19. Allowing for the uncertainty associated with the corrections, the collapse is quite reasonable, especially for $k_{1}^{*} \gtrsim 0.01$ with evidence of a small variation between 


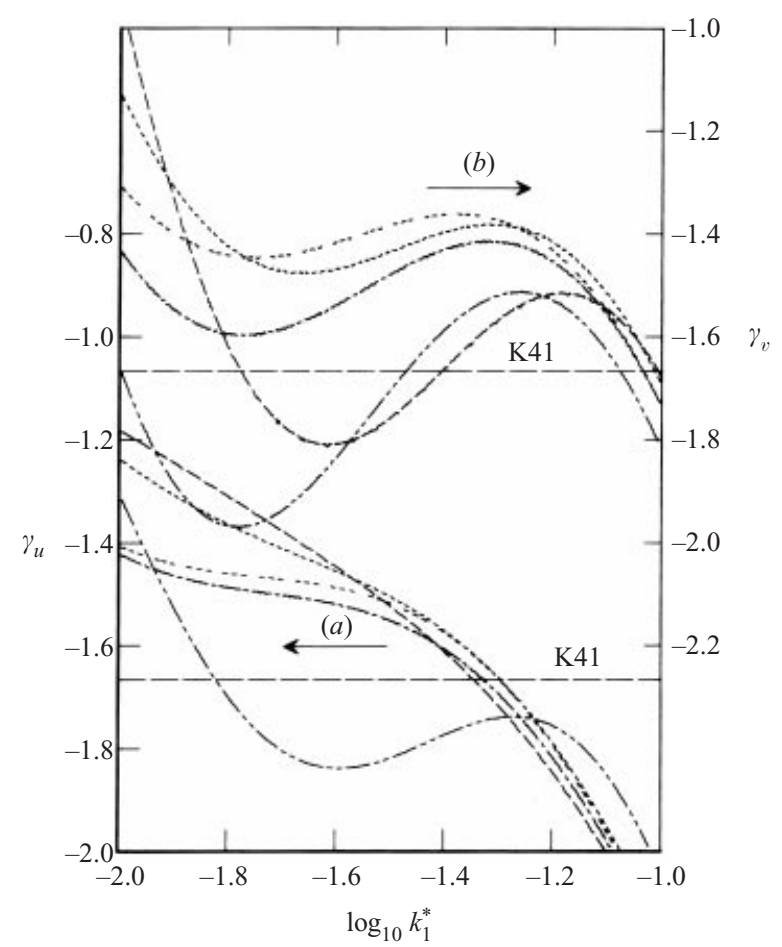

FIGURE 18. Distributions for each wake of $\gamma_{\alpha}$, the derivative with respect to $\log _{10} k_{1}^{*}$ of $\log _{10} \phi_{\alpha}^{*}\left(k_{1}^{*}\right)$. $R_{\lambda} \simeq 200$. Line types are as in figure 3. (a) $\gamma_{u} ;(b) \gamma_{v}$.

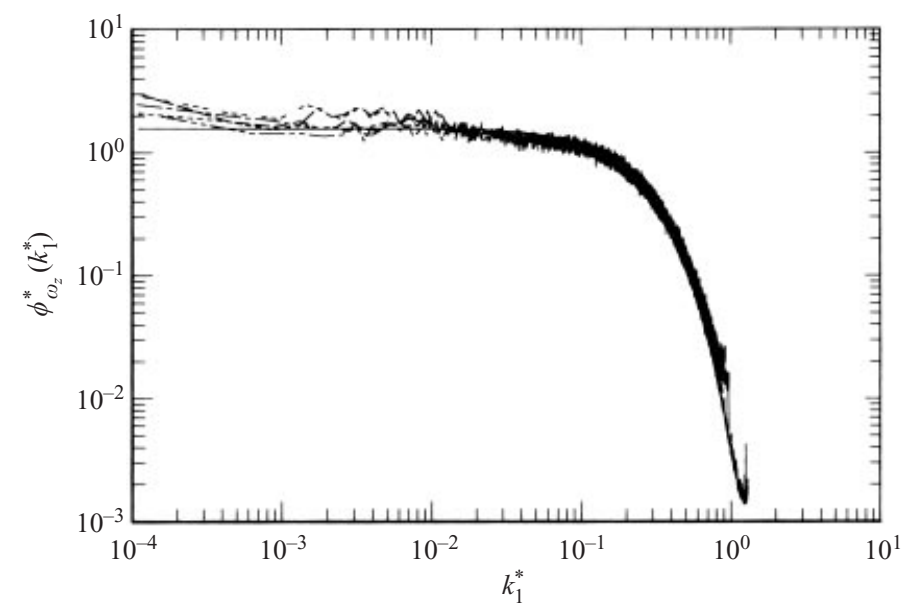

FIGURE 19. Kolmogorov-normalized spectra of $\omega_{z}$ at the same $R_{\lambda}(\simeq 200)$ in each of the wakes. Line types are as in figure 3.

the different wakes at smaller wavenumbers. The isotropic calculation of the vorticity spectrum (e.g. Van Atta 1991; Kim \& Antonia 1993)

$$
\phi_{\omega_{z}}^{c a l}\left(k_{1}\right)=\frac{5}{2} \phi_{\partial u / \partial x}\left(k_{1}\right)-\frac{k_{1}}{2} \frac{\partial \phi_{\partial u / \partial x}\left(k_{1}\right)}{\partial k_{1}}+2 \int_{k_{1}}^{\infty} \frac{\phi_{\partial u / \partial x}(k)}{k} \mathrm{~d} k
$$

is also shown in figure 19 for comparison with the corrected vorticity spectra. The comparison indicates that isotropy is satisfied for $k_{1}^{*} \gtrsim 0.01$ while the departure 


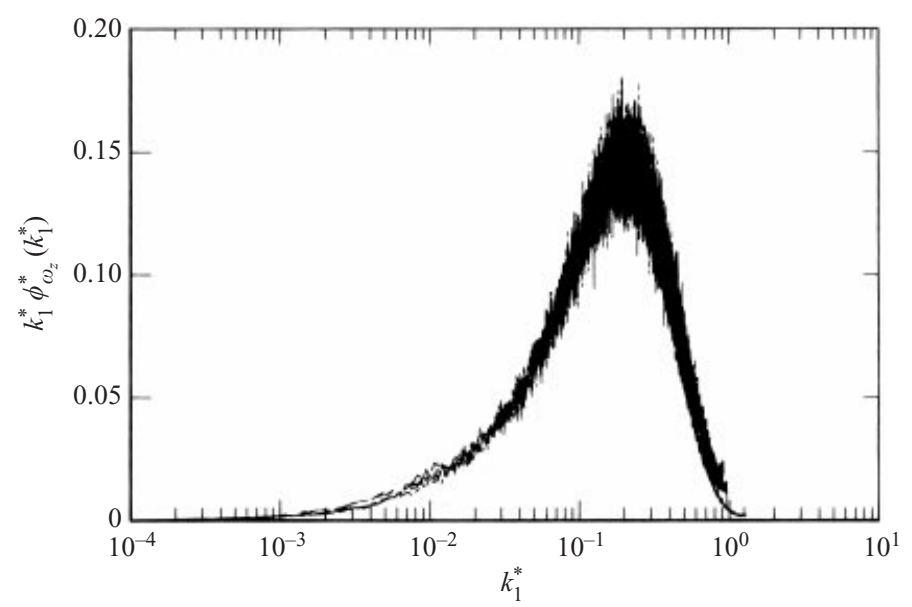

FIGURE 20. Kolmogorov-normalized spectra of $\omega_{z}$ multiplied by $k_{1}^{*}$ and shown on linear-log scales. Line types are as in figure 3.

between measurement and calculation in the range $k_{1}^{*} \leqslant 0.01$ reflects different degrees of departure from isotropy. The presentation in figure 20 , where $k_{1}^{*} \phi_{\omega_{z}}^{*}\left(k_{1}^{*}\right)$ is plotted on a linear scale against $\log k_{1}^{*}$, allows wavenumbers which contribute most to $\left\langle\omega_{z}^{* 2}\right\rangle$ to be identified. Accordingly, the peak in the distribution occurs near $k_{1}^{*} \simeq 0.2$ as was observed in the simulations of She et al. (1993) and the measurements of Antonia et al. (1996). The latter authors suggested that a possible interpretation of the spectral peak is that the vortical structures which contribute most to the enstrophy translate with a frequency corresponding to $k_{1}^{*} \simeq 0.2$, providing the convection velocity $U_{c}$ of the structures is approximately equal to the local mean velocity. Differences in the convection velocity of the vortices are expected however, especially between the porous-body wakes (where $U_{c} \simeq U$ ) and the solid-body wakes where $U_{c}$ can be slightly larger than $U$ (cf. Zhou \& Antonia 1992, 1993, 1994). This variation in $U_{c}$ may be responsible for the 'fuzziness' near the peak in figure 20. Another interpretation of the peak is that the most intense structures have a characteristic dimension of about $5 \eta$; this value would be the same in all the wakes. The corrected vorticity variance $\left\langle\omega_{z}^{* 2}\right\rangle$, obtained by integrating the corrected vorticity spectrum, i.e. $\int_{0}^{\infty} \phi_{\omega_{z}}^{*}\left(k_{1}^{*}\right) \mathrm{d} k_{1}^{*}=\left\langle\omega_{z}^{* 2}\right\rangle$, is plotted in figure 21 against $R_{\lambda}$. The values are nearly equal in all the wakes and approximately independent of $R_{\lambda}$. In homogeneous turbulence, the Kolmogorov-normalized enstrophy must be equal to 1 since $\langle\epsilon\rangle=v\left\langle\omega^{2}\right\rangle$. If local isotropy is assumed, $\left\langle\omega_{x}^{2}\right\rangle=\left\langle\omega_{y}^{2}\right\rangle=\left\langle\omega_{z}^{2}\right\rangle$ so that $\left\langle\omega_{z}^{* 2}\right\rangle=1 / 3$. The values shown in figure 21 are, on average, about $7 \%$ larger than $1 / 3$. It should be recalled however that $\langle\epsilon\rangle_{\text {iso }}$ has been used and it may be speculated that it underestimates the true mean energy dissipation rate, thus accounting for the observed inequality $\left\langle\omega_{z}^{* 2}\right\rangle>1 / 3$. Some support for this speculation is provided by values of the ratio $\left\langle(\partial v / \partial x)^{2}\right\rangle /\left\langle(\partial u / \partial x)^{2}\right\rangle$, which are, typically, about $15 \%$ larger than the isotropic value of 2 . However, the ratio $\left\langle(\partial u / \partial y)^{2}\right\rangle /\left\langle(\partial u / \partial x)^{2}\right\rangle$ is typically about $20 \%$ smaller than 2 . A definite assessment cannot therefore be made with respect to whether $\langle\epsilon\rangle$ is indeed greater than $\langle\epsilon\rangle_{\text {iso }}$ on the basis of the limited number of components of $\langle\epsilon\rangle$ than are available to us.

Distributions of $p_{\omega_{z}}$, the p.d.f. of $\omega_{z}$ where $\int_{-\infty}^{\infty} p_{\omega_{z}} \mathrm{~d} \omega_{z}=1$ are shown in figure 22, with $\omega_{z}$ normalized by its r.m.s. value. The collapse among the different wakes (it is not possible to distinguish between different lines in figure 22) is consistent with the 


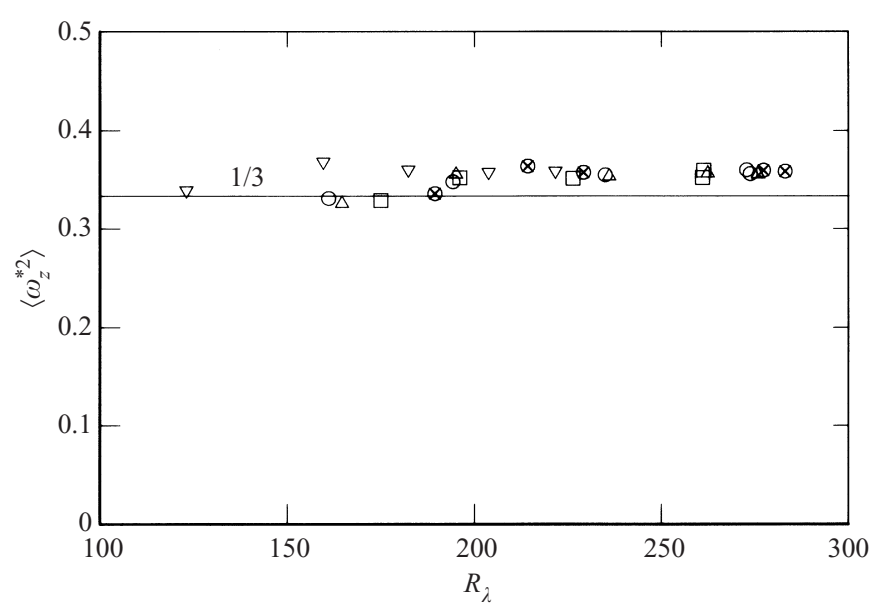

FIGURE 21. Kolmogorov-normalized variance of $\omega_{z}$. Symbols are as in figure 2.

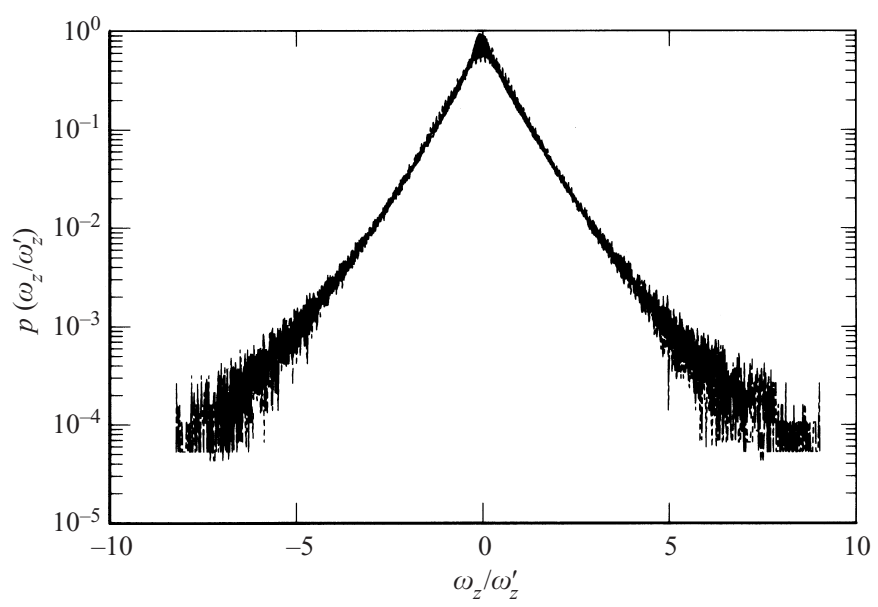

FIGURE 22. Probability density function of $\omega_{z}$. Line types are as in figure 3 .

collapse of the $\omega_{z}$ spectra (figures 20,21). It is also consistent with the constancy of $R_{\lambda}$. Antonia \& Zhou (2000) presented measurements, obtained in several flows, which showed an evolution of $p\left(\omega_{z}\right)$ with $R_{\lambda}$. The tails of the p.d.f. spread out further as $R_{\lambda}$ increased whereas the shape contracted and the amplitude increased around $\omega_{z}=0$, suggesting an increase in intermittency as $R_{\lambda}$ increases. For the data considered by Antonia \& Zhou (2000), $\left\langle\omega_{z}^{* 2}\right\rangle$ was, as for the present data, independent of $R_{\lambda}$. Certainly, local isotropy imposes a constraint on the shape of the p.d.f., since $\int_{-\infty}^{\infty} \omega_{z}^{* 2} p\left(\omega_{z}^{*}\right) \mathrm{d}\left(\omega_{z}^{*}\right)$ should be equal to $1 / 3$, as it does in the case of the spectrum. It is nonetheless interesting that, unlike the velocity spectra or velocity structure functions, the spectra (and p.d.f.) of $\omega_{z}$ appear not to be affected in any significant way by the differences in large-scale organization between the various wakes. A perfect collapse of $p\left(\omega_{z}\right)$ would imply that all moments of $\omega_{z}$ should be the same for each wake. The present data suggest that $F_{\omega_{z}}$, the flatness factor of $\omega_{z}$, is in fact slightly larger in the two porous-body wakes (10.5 and 10.9) than in the three solid-body wakes (7.6, 8.2 and 8.3). This in turn implies a more enhanced small-scale intermittency for the former than the latter flows. 


\section{Conclusions}

The present study was carried out mainly to provide information on the effect of initial conditions for nominally the 'same' flow (a two-dimensional turbulent wake) on the small-scale motion at effectively the same value $(\simeq 200)$ of $R_{\lambda}$, the Taylor microscale Reynolds number. Associated with each of the five different wakes were various degrees of large-scale organization and different levels of anisotropy, as measured by the ratios $\left\langle v^{2}\right\rangle /\left\langle u^{2}\right\rangle$ and $L_{v} / L_{u}$. Measurements were made with a spanwise vorticity probe on the wake centreline where the mean shear is zero and the flow is fully turbulent, so that there is no influence from the intermittency associated with the turbulent/non-turbulent interface.

In the dissipative range, the distributions of $\left\langle\left(\delta v^{*}\right)^{2}\right\rangle$ do not appear to collapse as well as those for $\left\langle\left(\delta u^{*}\right)^{2}\right\rangle$. This is partly due to the use of the relation $\langle\epsilon\rangle_{\text {iso }}=$ $15 v\left\langle(\partial u / \partial x)^{2}\right\rangle$ which forces the collapse of $\left\langle\left(\delta u^{*}\right)^{2}\right\rangle$ at small $r^{*}$. The derivative variances indicate departures from isotropy in each wake. The variance $\left\langle(\partial v / \partial x)^{2}\right\rangle$ is consistently greater than $\left\langle(\partial u / \partial x)^{2}\right\rangle$ in each wake but the magnitude of the excess differs slightly in each case, which is consistent with the imperfect collapse of $\left\langle\left(\delta v^{*}\right)^{2}\right\rangle$ at small $r^{*}$. There is a marked effect outside the dissipative range on both $\left\langle\left(\delta u^{*}\right)^{2}\right\rangle$ and, more especially, $\left\langle\left(\delta v^{*}\right)^{2}\right\rangle$. In particular, the distribution of $\left\langle\left(\delta v^{*}\right)^{2}\right\rangle$ is sensitive to the way energy is injected into the large scales. Associated with the significant changes in $\left\langle v^{2}\right\rangle /\left\langle u^{2}\right\rangle$ and $L_{v} / L_{u}$ between the different wakes are appreciable variations in both the shape and magnitude of $\left\langle\left(\delta v^{*}\right)^{2}\right\rangle$ over what can, at best, be tenuously described as the inertial (or scaling) range. Oscillations are observed beyond the upper end of this range for the porous-body wakes, consistent with the strong periodicity evident in the autocorrelations of $v$ for the screen-strip and screen-cylinder wakes. It is not possible to identify a range of $r^{*}$ for which the local derivatives of $\left\langle\left(\delta u^{*}\right)^{2}\right\rangle$ and $\left\langle\left(\delta v^{*}\right)^{2}\right\rangle$ may be considered to be constant, so that the concept of a K41 inertial range is strictly not valid since unique values of scaling exponents cannot be determined. The use of the ESS method provides estimates of scaling exponents which are 'relative' to $\left\langle|\delta u|^{3}\right\rangle$. These estimates indicate that the magnitude of the transverse exponent exceeds that of the longitudinal exponent in the porous-body wakes, an indication consistent with that inferred from the behaviour of the local slopes of the secondorder structure functions (figure 9) and the spectra (figure 19). The latter do not reflect the influence of the large-scale anisotropy outside the dissipative range as sensitively as the structure functions, apparently as a result of the different types of constraints that exist between spectra and structure functions. However, outside the dissipative range, the local slope of $\phi_{v}\left(k_{1}\right)$, like that of $\left\langle(\delta v)^{2}\right\rangle$, is steepest for the wake of the screen strip. This behaviour is consistent with the large values of $\left\langle v^{2}\right\rangle /\left\langle u^{2}\right\rangle$ and small values of $L_{v} / L_{u}$ in that flow. The magnitude of $C_{\epsilon}$, the mean energy dissipation rate parameter, is also smallest in this flow; in particular, the results of figures 2 and 5 indicate an inverse relationship between $C_{\epsilon}$ and the anisotropy of the large-scale motion, as measured by $\left\langle v^{2}\right\rangle /\left\langle u^{2}\right\rangle$ but also underline the dependence that exists, at least at moderate Reynolds numbers, between small and large scales. At the level of velocity derivative variances, there is little evidence to suggest that the departure from isotropy is different in the screen-strip wake than in the other wakes. However, the large magnitude and oscillatory behaviour of $\left\langle(\delta v)^{3}\right\rangle$ in the screen-strip wake (figure 11) highlights the strong anisotropy, observed in this flow, outside the dissipative range.

There is evidence, albeit limited, to suggest that the difference between longitudinal and transverse scaling exponents will, when $R_{\lambda}$ is fixed, depend on both $\left\langle v^{2}\right\rangle /\left\langle u^{2}\right\rangle$ and $L_{v} / L_{u}$. Such a dependence is consistent with the empirical model proposed by 
Romano \& Antonia (2001). When both $R_{\lambda}$ and $\left\langle v^{2}\right\rangle /\left\langle u^{2}\right\rangle$ are kept constant, as was the case for the screen and square-cylinder wakes, the difference between the exponents reflects the measured difference in the length scale ratio $L_{v} / L_{u}$.

The present results tend to suggest that the differences observed between the different wakes cannot simply be ascribed to whether the wake generator is solid or porous. Although the two porous body wakes are characterized by a relatively strong periodicity (e.g. figure 3), the significant differences observed between the screen-strip and screen-cylinder wakes suggest that the shape of the generator is also important.

On the basis of the present data, obtained at moderate $R_{\lambda}$, it is difficult to make any authoritative comment on whether small-scale turbulence becomes universal at very large $R_{\lambda}$. The data obtained over a much larger range of $R_{\lambda}$ (e.g. Pearson \& Antonia 2001) are not inconsistent with an approach towards such a state. However, the present data suggest that, for moderate- $R_{\lambda}$ flows, the properties of small-scale turbulence, especially those associated with a scaling range, depend not only on $R_{\lambda}$ but also on the way the energy is injected into the large scales. Figures 7 and 8 clearly indicate that the effect of initial conditions, which leads to different levels of large-scale anisotropy, is at least as important as that which occurs, in any particular flow, due to a variation in $R_{\lambda}$.

R. A. A. acknowledges the continuing support of the Australian Research Council.

\section{REFERENCES}

Anselmet, F., Gagne, Y., Hopfinger, E. J. \& Antonia, R. A. 1984 High order velocity structure functions in turbulent shear flows. J. Fluid Mech. 140, 63-89.

Antonia, R. A. \& Mi, J. 1998 Approach towards self-preservation of turbulent cylinder and screen wakes. Expl Thermal Fluid Sci. 17, 277-284.

Antonia, R. A., Romano, G. P. \& Orlandi, P. 1998 Scaling of longitudinal velocity increments in a fully developed turbulent channel flow. Phys. Fluids 10, 3239-3241.

Antonia, R. A. \& Pearson, B. R. 2000 Effect of initial conditions on the mean energy dissipation rate and the scaling exponent. Phys. Rev. E 62, 8086-8090.

Antonia, R. A., Pearson, B. R. \& Zhou, T. 2000 Reynolds number dependence of second-order velocity structure functions. Phys. Fluids 12, 3000-3006.

Antonia, R. A., Satyaprakash, B. R. \& Hussain, A. K. M. F. 1982 Statistics of fine scale velocity in turbulent plane and circular jets. J. Fluid Mech. 119, 55-89.

Antonia, R. A., Shafi, H. S. \& ZhU, Y. 1996 A note on the vorticity spectrum. Phys. Fluids 8, 2196-2202.

Antonia, R. A. \& Smalley, R. J. 2000 Velocity and temperature scaling in a rough wall boundary layer. Phys. Rev. E 61, 640-646.

Antonia, R. A. \& Zhou, T. 2000 Flow and Reynolds number dependencies of turbulent vorticity fluctuations. In Advances in Turbulence III (ed. C. Dopazo), pp. 801-804. Barcelona, CIMNE.

Antonia, R. A., Zhu, Y. \& Kim, J. 1993 On the measurement of lateral velocity derivatives in turbulent flows. Exps. Fluids 15, 65-69.

Arad, I., Biferale, L., Mazzitelli, I. \& Procaccia, I. 1999a Disentangling scaling properties in anisotropic and inhomogeneous turbulence. Phys. Rev. Lett. 82, 5040-5043.

Arad, I., Dhruva, B., Kurien, S., L'vov, V., Procaccia, I. \& Sreenivasan, K. R. 1998 The extraction of anisotropic contributions in turbulent flows. Phys. Rev. Lett. 81, 5330-5333.

Arad, I., L'vov, V. \& Procaccia, I. 1999b Correlation functions in isotropic and anisotropic turbulence: the role of the symmetry group. Phys. Rev. E 59, 6753-6765.

Benzi, R., Ciliberto, S., Tripiccione, R., Baudet, C., Massaioli, F. \& Succi, S. 1993 Extended self-similarity in turbulent flows. Phys. Rev. E 48, R29-R32.

Biferale, L. \& Toschi, F. 2001 Anisotropies in homogeneous turbulence: hierarchy of scaling exponents and intermittency of the anisotropic sectors. Phys. Rev. Lett. 86, 4831-4834. 
Biferale, L. \& Vergassola, M. 2001 Isotropy vs anisotropy in small-scale turbulence. Phys. Fluids 13, 2139-2141.

Browne, L. W. B., Antonia, R. A. \& Chua, L. P. 1989 Velocity vector cone angle in turbulent flows. Exps. Fluids 8, 13-16.

Cannon, S., Champagne, F. \& Glezer, A. 1993 Observations of large-scale structures in wakes behind axisymmetric bodies. Exps. Fluids 14, 447-450.

CASTRO, I. P. 1971 Wake characteristics of two-dimensional perforated plates normal to an air stream. J. Fluid Mech. 46, 599-609.

Chen, S., Sreenivasan, K. R., Nelkin, M. \& CaO, N. 1997 A refined similarity hypothesis for transverse structure functions. Phys. Rev. Lett. 79, 1253

Cimbala, J. M. \& Krein, M. V. 1990 Effect of freestream conditions on the far wake of a circular cylinder. AIAA J. 28, 1369-1373.

Cimbala, J. M., Nagib, H. M. \& Roshko, A. 1988 Large structure in the far wakes of twodimensional bluff bodies. J. Fluid Mech. 190, 265-298.

Cimbala, J. M. \& Park, W. J. 1990 An experimental investigation of the turbulent structure in a two-dimensional momentumless wake. J. Fluid Mech. 213, 479-509.

Danaila, L., Anselmet, F., Zhou, T. \& Antonia, R. A. 1999 A generalization of Yaglom's equation which accounts for the large-scale forcing in heated grid turbulence. J. Fluid Mech. 391, 359-372.

Danaila, L., Anselmet, F., Zhou, T. \& Antonia, R. A. 2001 Turbulent energy scale-budget equations in a fully developed channel flow. J. Fluid Mech. 430, 87-109.

Dhruva, B., Tsuji, Y. \& Sreenivasan, K. R. 1997 Transverse structure functions in high-Reynoldsnumber turbulence. Phys. Rev. E 56, R4928-R4930.

Frisch, U. 1995 Turbulence: The Legacy of A. N. Kolmogorov. Cambridge University Press.

Hou, T. Y., Wu, X-H., Chen, S. \& Zhou, Y. 1998 Effect of finite computational domain on turbulence scaling law in both physical and spectral spaces. Phys. Rev. E 58, 5841-5844.

HuAng, Z. \& KefFER, J. F. 1996 Development of structure within the turbulent wake of a porous body. Part 1. J. Fluid Mech. 323, 103-115.

Kim, J. \& AntoniA, R. A. 1993 Isotropy of the small-scales of turbulence at small Reynolds number. J. Fluid Mech. 251, 219-238.

Kolmogorov, A. N. 1941 a The local structure of turbulence in an incompressible fluid for very large Reynolds numbers. Dokl. Akad. Nauk. SSSR 30, 301-305.

Kolmogorov, A. N. 1941 bnergy dissipation in locally isotropic turbulence. Dokl. Akad. Nauk. SSSR 32, 16-18.

Kolmogorov, A. N. 1962 A refinement of previous hypotheses concerning the local structure of turbulence in a viscous incompressible fluid at high Reynolds number. J. Fluid Mech. 13, $82-85$.

Kurien, S. \& Sreenivasan, K. R. 2000 Anisotropic scaling contributions to high-order structure functions in high-Reynolds-number turbulence. Phys. Rev. E 62, 2206-2212.

Mydlarski, L. \& Warhaft, Z. 1986 On the onset of high-Reynolds-number grid-generated wind tunnel turbulence. J. Fluid Mech. 320, 331-368.

Oвukhov, A. M. 1962 Some specific features of atmospheric turbulence. J. Fluid Mech. 13, 77-81.

Papailiou, D. D. \& LyKoudis, P. S. 1974 Turbulent vortex and the entrainment mechanism of the turbulent wake. J. Fluid Mech. 62, 11-31.

PARK, W. J. \& Cimbala, J. M. 1991 The effect of jet injection geometry on two-dimensional momentumless wakes. J. Fluid Mech. 224, 29-47.

Pearson, B. R. 1999 Some experiments on small scale turbulence. PhD Thesis, University of Newcastle, Australia.

Pearson, B. R. \& Antonia, R. A. 2001 Reynolds-number dependence of turbulent velocity and pressure increments. J. Fluid Mech. 444, 343-382.

Romano, G. P. \& Antonia, R. A. 2001 Longitudinal and transverse structure functions in a turbulent round jet: effect of initial conditions and Reynolds number. J. Fluid Mech. 436, 231-248.

She, Z.-S., Chen, S., Doolen, G., Kraichnan, R. N. \& Orszag, S. A. 1993 Reynolds number dependence of isotropic Navier-Stokes turbulence. Phys. Rev. Lett. 70, 3251-3254.

SHE, S. Z. \& LeVEQUE, E. 1994 Universal scaling laws in fully developed turbulence. Phys. Rev. Lett. 72, 336-339. 
Sreenivasan, K. R. 1981 Approach to self-preservation in plane turbulent wakes. AIAA J. 19, 1365-1367.

Sreenivasan, K. R. 1995 The energy dissipation in turbulent shear flows. In Symposium on Developments in Fluid Dynamics and Aerospace Engineering (ed. S. M. Deshpande, A. Prabhu, K. R. Sreenivasan and P. R. Viswanath), pp. 159-190. Bangalore: Interline Publishers.

Sreenivasan, K. R. 1996 The passive scalar spectrum and the Obukhov-Corrsin constant. Phys. Fluids 8, 189-196.

Sreenivasan, K. R. 1999 Fluid turbulence. Rev. Mod. Phys. 71, S383-S395.

Sreenivasan, K. R. \& Antonia, R. A. 1997 The phenomenology of small-scale turbulence. Annu. Rev. Fluid Mech. 29, 435-472.

Sreenivasan, K. R. \& Dhruva, B. 1998 Is there scaling in high-Reynolds-number turbulence? Prog. Theor. Phys. Supp. 130, 103-120.

Sreenivasan, K. R. \& Kailasnath, P. 1993 An update on the intermittency exponent in turbulence. Phys. Fluids A 5, 512-514.

Tennekes, H. \& Lumley, J. L. 1972 A First Course in Turbulence. MIT Press.

VAN AtTA, C. W. 1991 Local isotropy of the smallest scales of turbulent scalar and velocity fields. Proc. R. Soc. Lond. A 434, 139-137.

Vincent, A. \& MenEguzZI, M. 1991 The spatial structure and statistical properties of homogneeous turbulence. J. Fluid Mech. 225, 1-20.

Zhou, T. \& Antonia, R. A. 2000 Reynolds number dependence of the small scale structure of grid turbulence. J. Fluid Mech. 406, 81-107.

Zhou, Y. \& Antonia, R. A. 1992 Convection velocity measurements in cylinder wake. Exps. Fluids 13, 63-70.

Zhou, Y. \& Antonia, R. A. 1993 A study of turbulent vortices in the near-wake of a cylinder. J. Fluid Mech. 253, 643-661.

Zhou, Y. \& Antonia, R. A. 1994 Effect of initial conditions on vortices in a turbulent near wake. AIAA J. 32, 1207-1213.

Zhou, Y. \& Antonia, R. A. 1995 Memory effects in a turbulent plane wake. Exps. Fluids 19, $112-120$.

Zhu, Y. \& Antonia, R. A. 1995 Effect of wire separation in X-probe measurements in a turbulent flow. J. Fluid Mech. 287, 199-223. 